\title{
Moiré patterns between aperiodic layers: quantitative analysis and synthesis
}

\author{
Isaac Amidror \\ Laboratoire de Systèmes Périphériques, Ecole Polytechnique Fédérale de Lausanne, 1015 Lausanne, Switzerland
}

Received March 7, 2003; accepted June 5, 2003

\begin{abstract}
Moiré effects that occur in the superposition of aperiodic layers such as random dot screens are known as Glass patterns. Unlike classical moiré effects between periodic layers, which are periodically repeated throughout the superposition, a Glass pattern is concentrated around a certain point in the superposition, and farther away from this point it fades out and disappears. I show that Glass patterns between aperiodic layers can be analyzed by using an extension of the Fourier-based theory that governs the classical moire patterns between periodic layers. Surprisingly, even spectral-domain considerations can be extended in a natural way to aperiodic cases, with some straightforward adaptations. These new results allow us to predict quantitatively the intensity profile of Glass patterns; furthermore, they open the way to the synthesis of Glass patterns that have any desired shapes and intensity profiles. (c) 2003 Optical Society of America
\end{abstract}

OCIS codes: $070.0070,120.4120$.

\section{INTRODUCTION}

When two identical random dot screens, or any two identical aperiodic structures, are superposed on top of each other with a small angle or scaling difference, a typical moiré effect [Fig. 1(a)] appears in the superposition. This moiré effect is known in the literature as a Glass pattern, after Leon Glass, who described it in the late 1960s. ${ }^{1,2}$ Unlike a moiré effect between periodic dot screens [Fig. 1(b)], which is periodic and extends throughout the entire superposition, ${ }^{3}$ a Glass pattern is concentrated around a certain point in the superposition, and it gradually fades out and disappears as we move farther away from this point.

In all of the Glass patterns studied in the past, the two superposed random layers were either identical or slightly transformed (scaled, rotated, or translated) copies of each other. As widely reported in literature, the resulting Glass patterns in such cases have a typical shape resembling a top-viewed funnel or a distant galaxy in the night sky. However, it was recently discovered ${ }^{4}$ that by choosing appropriate dot shapes for the dots of the two superposed aperiodic screens, one may obtain in the superposition a Glass pattern of any desired shape and intensity profile, as illustrated in Fig. 2. The aim of the present contribution is to provide a full qualitative and quantitative theoretical explanation of Glass patterns in general and of this surprising phenomenon in particular. As we will see, the explanation is based on an extension into the aperiodic case of results that are already known for periodic dot screens. But whereas in the superposition of periodic dot screens the moire profile is periodically repeated throughout the superposition, in the present case the Glass pattern obtained in the superposition consists of only one moiré profile (compare Figs. 2 and 3 ).

Our approach here is based on a Fourier-based analysis of the behavior of Glass patterns both in the image do- main and in the spectral domain. We start in Section 2 with a review of the basic concepts from the periodic case that are required for understanding the rest of this paper. In Section 3 we investigate the superposition of aperiodic line gratings, and then, using these results, we proceed in Section 4 to the case of aperiodic screens. In both cases we analyze the resulting Glass patterns and their intensity profiles both in the image domain and in the Fourier domain as a generalization of the moiré theory between periodic layers. This will also show us how to synthesize Glass patterns that have any desired intensity profiles and shapes. Then, in Section 5, an explanation is given of why in the superposition of aperiodic layers no higherorder moirés may exist, unlike in the periodic case. Finally, in Section 6 the main conclusions of the paper are presented.

Remark: The PostScript files that generate the dot screens and the line gratings used in the figures of this article are available on the internet. ${ }^{5}$ They can be downloaded and printed on transparencies with any standard PostScript printer. Superposing these transparencies manually with varying orientations, shifts, etc., can give a vivid demonstration of the Glass (or moiré) patterns and their dynamic behavior in the superposition, even beyond the few static figures that illustrate this paper.

\section{BACKGROUND AND BASIC CONCEPTS}

The rules that govern the qualitative behavior of Glass patterns in the superposition of general aperiodic layers have been investigated in previous contributions. ${ }^{1,2,6,7}$ However, none of the previous studies provides a quantitative analysis of these phenomena and of their intensity profiles. In the present study we will investigate the layer superpositions and the resulting Glass patterns by using a Fourier-based approach, which is a direct extension of the theory that governs the superposition of periodic layers. This powerful mathematical approach will 


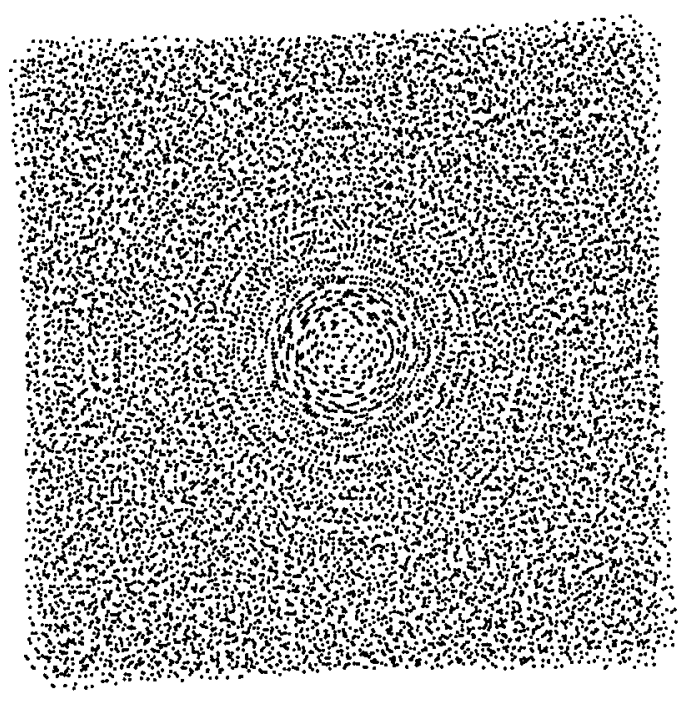

(a)

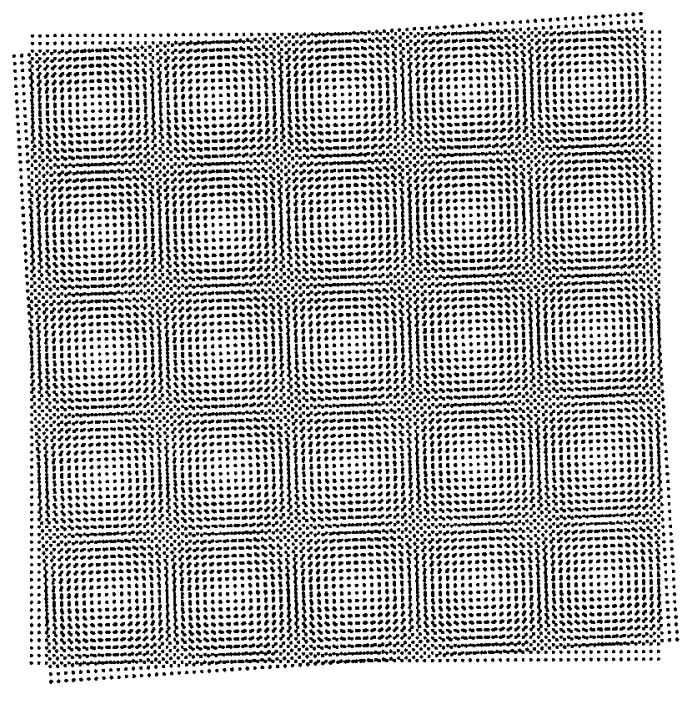

(b)

Fig. 1. (a) Superposition of two identical aperiodic dot screens with a small angle difference gives a moiré effect in the form of a Glass pattern around the center of rotation. (b) When the superposed layers are periodic, a Glass pattern is still generated around the center of rotation, but owing to the periodicity of the layers, this pattern is periodically repeated throughout the superposition, thus generating a periodic moiré pattern.

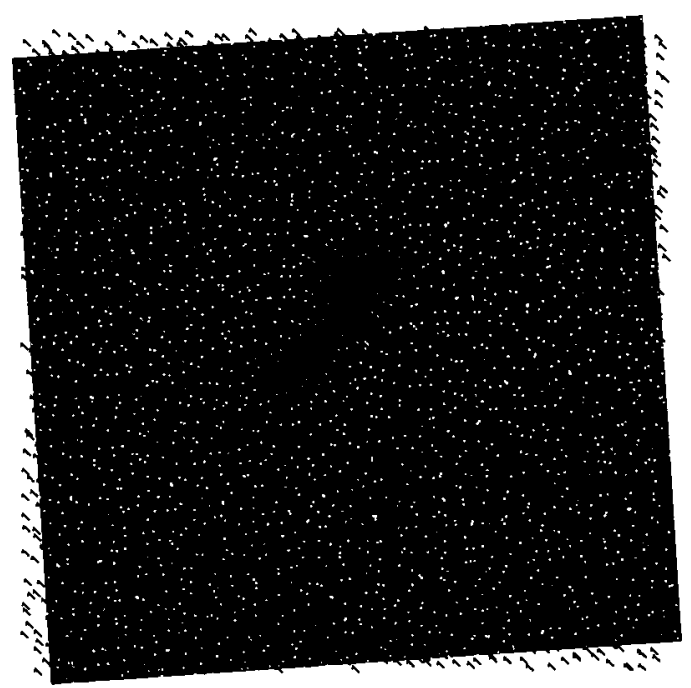

Fig. 2. Superposition with a small angle difference of a random dot screen consisting of 1 -shaped dots and a random dot screen consisting of small white dots (pinholes) on a black background, where the dot locations in both screens are identical, gives a single 1-shaped moiré intensity profile (Glass pattern).

also allow us to predict quantitatively the intensity profile of the resulting Glass patterns, and, furthermore, it will lead us to a method for synthesizing Glass patterns that have any desired shapes and intensity profiles.

Before we proceed to our new results, we review in the rest of this section the basic concepts and terminology that are required for understanding the next sections.

\section{A. General Properties of Superposed Layers and of Their Fourier Spectra}

First of all, let us mention that throughout this paper we are concerned only with monochrome, black-and-white images (or layers). This means that each image can be

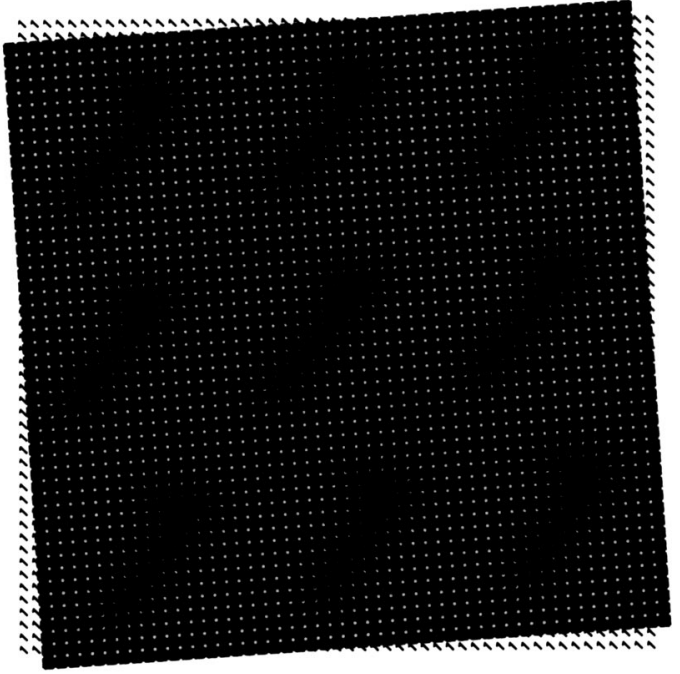

Fig. 3. Periodic counterpart: The superposition with a small angle difference of a periodic dot screen consisting of 1-shaped dots and a periodic dot screen consisting of small white dots (pinholes) on a black background gives a periodic 1-shaped moiré intensity profile.

represented by a reflectance function, which assigns to any point $(x, y)$ of the image a value between 0 and 1 representing its light reflectance: 0 for black (i.e., no reflected light), 1 for white (i.e., full light reflectance), and intermediate values for in-between shades. In the case of transparencies, the reflectance function is replaced by a transmittance function defined in a similar way. The superposition of such images can be done by overprinting or by laying printed transparencies on top of each other. Since the superposition of black and any other shade always gives black, this suggests a multiplicative model for the superposition of monochrome images. Thus when $m$ monochrome images are superposed, the reflectance of 
the resulting image is given by the product of the reflectance functions of the individual images:

$$
r(x, y)=r_{1}(x, y) r_{2}(x, y) \ldots r_{m}(x, y) .
$$

According to the convolution theorem (Ref. 8, p. 244), the Fourier transform of the product function is the convolution of the Fourier transforms of the individual functions. Therefore, if we denote the Fourier transform of each function by the respective capital letter and the twodimensional (2D) convolution by **, the spectrum of the superposition is given by

$$
R(u, v)=R_{1}(u, v) * * R_{2}(u, v) * * \cdots * * R_{m}(u, v) .
$$

\section{B. Spectra of Periodic and Aperiodic Layers}

Although the structures that we study in the present paper are not periodic, it would be helpful to start with a short reminder from the periodic case, whose mathematical behavior is already fully understood. ${ }^{3}$ Suppose we are given a periodic image defined on the continuous $(x, y)$ plane, such as a line grating or a dot screen. The spectrum of a periodic image on the $(u, v)$ plane is not continuous but rather consists of impulses corresponding to the frequencies that appear in the Fourier series decomposition of the image. In the case of a onefold periodic image, such as a line grating, the spectrum consists of a one-dimensional (1D) "comb" of impulses centered about the origin; in the case of a twofold periodic image, the spectrum is a 2D "nailbed" of impulses centered about the origin. Note that we sometimes use the more general term "cluster" for a comb or a nailbed.

Each impulse in the $2 \mathrm{D}$ spectrum is characterized by its geometric location and its amplitude. The geometric location of an impulse is represented by a frequency vector $\mathbf{f}$ in the spectrum plane, which connects the spectrum origin to the geometric location of the impulse. This vector can be expressed by its polar coordinates $(f, \theta)$, where $\theta$ is the direction of the impulse and $f$ is its distance from the origin (i.e., its frequency in that direction). In terms of the original image, the geometric location of an impulse in the spectrum determines the frequency $f$ and the direction $\theta$ of the corresponding periodic component in the image, and the amplitude of the impulse represents the intensity of that periodic component in the image. (Note that if the original image is not symmetric about the origin, the amplitude of each impulse in the spectrum may also have a nonzero imaginary component).

However, the question of whether an impulse in the spectrum represents a visible periodic component in the image depends strongly on properties of the human visual system. The fact that the eye cannot distinguish fine details above a certain frequency (i.e., below a certain period) suggests that the human visual system model includes a low-pass filtering stage. For the sake of simplicity, this low-pass filter can be approximated by the visibility circle, a circular step function around the spectrum origin whose radius represents the cutoff frequency (i.e., the threshold frequency beyond which fine detail is no longer detected by the eye). Obviously, the radius of the visibility circle depends on several factors such as the contrast of the observed details, the viewing distance, and light conditions. If the frequencies of the original image elements are beyond the border of the visibility circle in the spectrum, the eye can no longer see them; but if a strong enough impulse in the spectrum of the image superposition falls inside the visibility circle, then a moiré effect becomes visible in the superposed image.

Finally, a word about the Fourier spectra of aperiodic layers: Unlike the spectra of periodic layers, which are purely impulsive, the Fourier transform of an aperiodic layer is basically continuous. For example, the Fourier transform of a unit cube is a 2D sinc function; see Ref. 9, pp. 150-151. But when the layer's structure is very complex, as in the case of a random dot screen, its Fourier spectrum becomes very jumpy or noisy and presents a typical diffuse appearance (see Ref. 9, pp. 586-590; 600601).

\section{Moiré Effects in the Superposition of Periodic Gratings}

The simplest moire effects occur in the superposition of two straight periodic gratings, as shown in Fig. 4. Let $r_{1}(x, y)$ and $r_{2}(x, y)$ be such periodic gratings; their frequencies and orientations are given by their frequency vectors $\mathbf{f}_{1}$ and $\mathbf{f}_{2}$, respectively. The spectrum $R_{i}(u, v)$ of each of the original gratings consists of a comb whose impulses are located at integer multiples of the fundamental frequency $n \mathbf{f}_{i}$ :

$$
\begin{aligned}
& R_{1}(u, v)=\sum_{n=-\infty}^{\infty} a_{n}^{(1)} \delta_{n \mathbf{f}_{1}}(u, v), \\
& R_{2}(u, v)=\sum_{n=-\infty}^{\infty} a_{n}^{(2)} \delta_{n \mathbf{f}_{2}}(u, v) .
\end{aligned}
$$

Here, $\delta_{n \mathbf{f}_{i}}(u, v)$ denotes an impulse located in the spectrum at the frequency vector $n \mathbf{f}_{i}$, and $a_{n}^{(i)}$ is its amplitude.

When we superpose (i.e., multiply) the line gratings $r_{1}(x, y)$ and $r_{2}(x, y)$, the spectrum of the superposition is, according to the convolution theorem, the convolution of the two original combs, $R_{1}(u, v) * * R_{2}(u, v)$, which gives an oblique nailbed of impulses [see Fig. 4(f)]. This convolution of combs can be seen as an operation in which frequency vectors from the individual spectra are added vectorially, while the corresponding impulse amplitudes are multiplied. We can therefore express the geometric location of the general $\left(k_{1}, k_{2}\right)$ impulse in the spectrum convolution by the vectorial sum

$$
\mathbf{f}_{k_{1}, k_{2}}=k_{1} \mathbf{f}_{1}+k_{2} \mathbf{f}_{2}
$$

and its amplitude by

$$
a_{k_{1}, k_{2}}=a_{k_{1}}^{(1)} a_{k_{2}}^{(2)},
$$

where $\mathbf{f}_{i}$ denotes the frequency vector of the fundamental impulse in the spectrum of the $i$ th grating and $k_{i} \mathbf{f}_{i}$ and $a_{k_{i}}^{(i)}$ are, respectively, the frequency vector and the amplitude of the $k_{i}$ th harmonic impulse in the spectrum of the $i$ th grating.

The vectorial sum of Eq. (3) can also be written in terms of its Cartesian components. If $f_{i}$ are the frequencies of the original gratings and $\theta_{i}$ are the angles that 

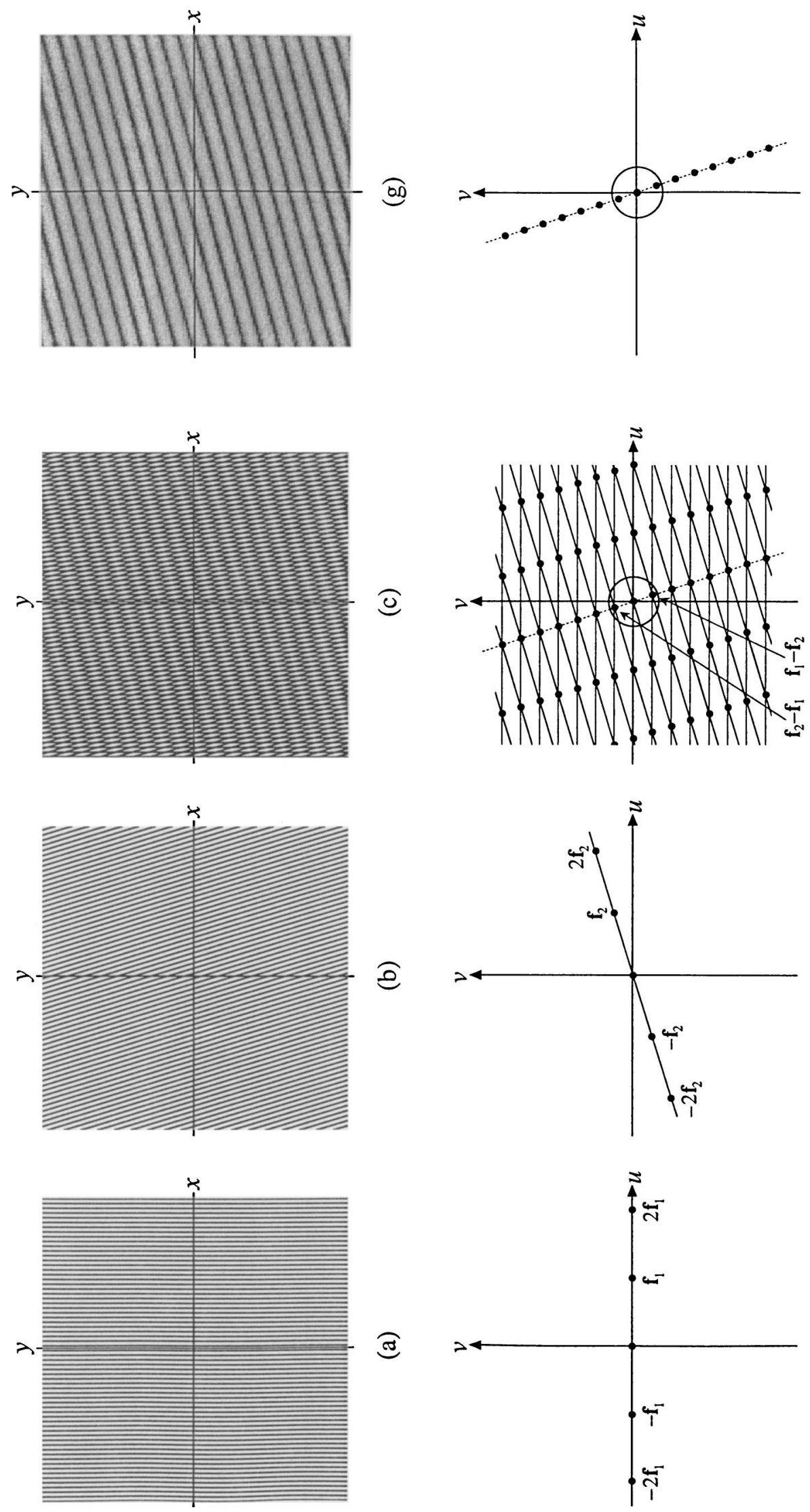

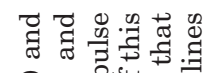

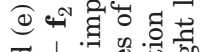
क्षै

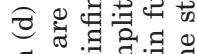
.

ᄅ

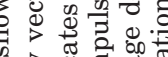

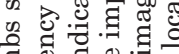

ర్

屯.

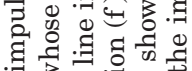

(1) 8 . .

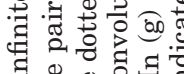

. $ه$ ه

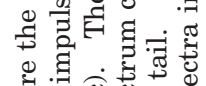

离.

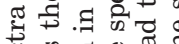

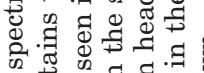

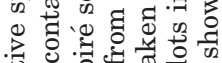

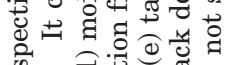

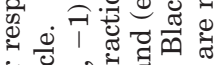

:

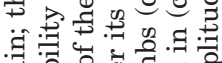

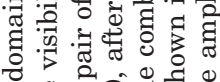

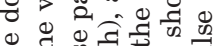

$\infty$ क⿺⿻一⿰冫⿰亅⿱丿丶丶⿱⿰㇒一乂

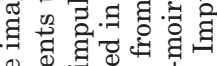

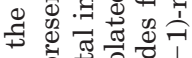

घ

()

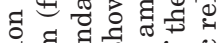

(ิ)

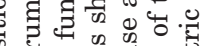

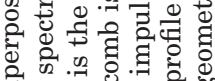

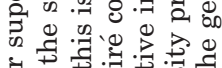

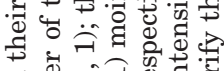

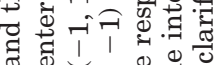

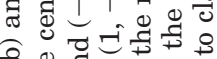

(2)

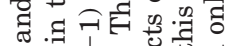

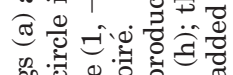

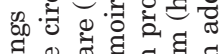

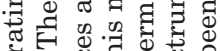

का

ఫ्?

छ $\dot{\theta}$.

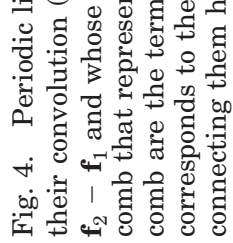


they form with the positive horizontal axis, then the coordinates $\left(f_{u}, f_{v}\right)$ of the $\left(k_{1}, k_{2}\right)$ impulse in the spectrum convolution are given by

$$
\begin{aligned}
& f u_{k_{1}, k_{2}}=k_{1} f_{1} \cos \theta_{1}+k_{2} f_{2} \cos \theta_{2}, \\
& f v_{k_{1}, k_{2}}=k_{1} f_{1} \sin \theta_{1}+k_{2} f_{2} \sin \theta_{2} .
\end{aligned}
$$

Therefore the frequency, the period, and the angle of the considered impulse are given by the length and the direction of the vector $\mathbf{f}_{k_{1}, k_{2}}$ as follows:

$$
f=\sqrt{f_{u}^{2}+f_{v}^{2}}, \quad T=1 / f, \quad \varphi=\arctan \left(f_{v} / f_{u}\right) .
$$

Now, if one of the new impulses in the spectrum convolution, say, the $\left(k_{1}, k_{2}\right)$ impulse, falls (together with its symmetric twin) close to the origin inside the visibility circle, this implies the existence in the superposed image of a moiré effect with a visible period. This moiré is represented in the spectrum convolution by a full comb of impulses, centered on the origin, that contains the $\left(k_{1}, k_{2}\right)$ impulse as well as all its harmonics. We call this moiré a $\left(k_{1}, k_{2}\right)$ moiré since the fundamental impulse of its comb is the $\left(k_{1}, k_{2}\right)$ impulse of the spectrum convolution. For example, in the case of Fig. $4(\mathrm{f})$, the $(1,-1)$ impulse [as well as its symmetric $(-1,1)$ impulse] falls inside the visibility circle; this indicates that the moire effect that is clearly visible in the grating superposition [Fig. 4(c)] is the first-order $(1,-1)$ moiré. The frequency and the period of this moiré can be found from Eqs. (5) and (6) by using $k_{1}=1$ and $k_{2}=-1$; equivalently, they can be found by a simple geometric consideration, as shown in Fig. 5 for the case with $f_{1}=f_{2}$ :

$$
\sin (\alpha / 2)=\frac{f_{M} / 2}{f}
$$

hence

$$
f_{M}=2 f \sin (\alpha / 2)
$$

and thus

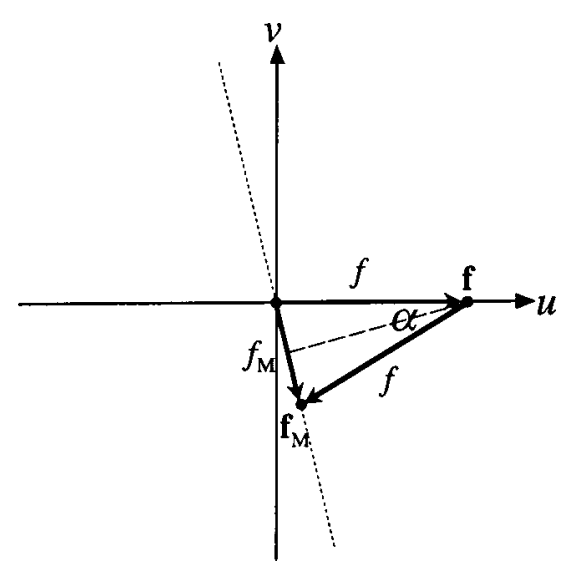

Fig. 5. Geometric consideration in the spectral domain for finding the frequency $f_{M}$ and the period $T_{M}$ of the $(1,-1)$ moire effect between two gratings with identical frequencies $f$ and an angle difference of $\alpha$. The dotted line indicates the infinite impulse comb that represents the $(1,-1)$ moiré [see Fig. 4(f)]; the fundamental impulse of this moiré has the frequency $f_{M}$.

$$
T_{M}=\frac{1}{2 f \sin (\alpha / 2)}=\frac{T}{2 \sin (\alpha / 2)} .
$$

The orientation of the moiré in this case is perpendicular to the bisector of the original gratings.

These equations can be generalized to the case in which the original gratings have different frequencies $f_{1} \neq f_{2}$; in this case the respective formulas are (see Ref. 3, p. 409, or Ref. 10)

$$
f_{M}=\left(f_{1}^{2}-2 f_{1} f_{2} \cos \alpha+{f_{2}}^{2}\right)^{1 / 2},
$$

and thus

$$
T_{M}=\frac{T_{1} T_{2}}{\left(T_{1}{ }^{2}+T_{2}{ }^{2}-2 T_{1} T_{2} \cos \alpha\right)^{1 / 2}},
$$

and the moiré orientation is

$$
\varphi_{M}=\arctan \left(\frac{T_{2} \sin \theta_{1}-T_{1} \sin \theta_{2}}{T_{2} \cos \theta_{1}-T_{1} \cos \theta_{2}}\right) .
$$

Returning to the general $\left(k_{1}, k_{2}\right)$ moiré in the superposition of two gratings, the location $\mathbf{f}_{k_{1}, k_{2}}$ of its fundamental impulse in the spectrum is given by Eq. (3). The $n$th impulse of the comb of this moire is the $\left(n k_{1}, n k_{2}\right)$ impulse in the spectrum convolution; its location is $n \mathbf{f}_{k_{1}, k_{2}}$, and its amplitude $d_{n}$ is given by $d_{n}=a_{n k_{1}, n k_{2}}$, which means, according to Eq. (4),

$$
d_{n}=a_{n k_{1}}^{(1)} a_{n k_{2}}^{(2)},
$$

where $a_{i}^{(1)}$ and $a_{i}^{(2)}$ are the respective impulse amplitudes from the combs of the first and the second line gratings. For example, in the case of the simplest first-order moire between two gratings, the $(1,-1)$ moiré [see Fig. 4(f)], the amplitudes of the moiré-comb impulses are given by

$$
d_{n}=a_{n}^{(1)} a_{-n}^{(2)} .
$$

In other words, we can say the following:

Proposition 1. The impulse amplitudes of the comb of the $(1,-1)$ moiré in the spectrum convolution are obtained by a term-by-term multiplication of the combs of the original superposed gratings, one of which being inverted (rotated by $180^{\circ}$ ) before the multiplication.

Since we already know the location of the impulses of the moiré comb, this comb can be considered, in fact, to be a product of the two original combs after they have been normalized (rotated and stretched) to fit the impulse locations of the resulting moiré comb. This moiré comb can be easily extracted from the spectrum convolution, as shown in Fig. 4(h). Thus by taking its inverse Fourier transform, we can reconstruct, back in the image domain, the isolated contribution of the moire in question to the image superposition; this is the intensity profile of the moiré [see Fig. 4(g)]. Note that although this moiré is visible both in the layer superposition [Fig. 4(c)] and in the extracted moiré intensity profile [Fig. 4(g)], the latter does not contain the fine structure of the original layers $r_{1}(x, y)$ and $r_{2}(x, y)$ but only the pure contribution of the extracted moiré itself.

However, this term-by-term multiplication of the original combs, as defined by Eq. (13), can be also interpreted, back in the image domain, by using the $T$-convolution 
theorem, which is the periodic counterpart of the convolution theorem. The full details can be found in Sec. 4.2 of Ref. 3; here we will only give the following result for the $(1,-1)$ moiré (as an illustration, refer to Fig. 4).

Proposition 2. The intensity profile of the $(1,-1)$ moire that is generated in the superposition of two periodic line gratings with periods $T_{1}$ and $T_{2}$ and an angle difference $\alpha$ can be seen from the image-domain point of view as the result of a three-stage process:

1. Normalization of the original gratings (by linear stretching and rotation transformations) in order to bring both of them to a common period and orientation (that is, to make their periods, or their impulse combs in the spectrum, coincide).

2. $T$ convolution of the two normalized line gratings.

3. Stretching and rotating the resulting normalized moiré intensity profile into its actual scale and orientation, as determined by Eqs. (10) and (11).

Thus, whereas the period and the orientation of the $(1,-1)$ moiré bands are determined by Eqs. (10) and (11), their intensity profile is governed by Proposition 2 . Note that in the particular case where $T_{1}=T_{2}$ and $\theta_{1} \approx \theta_{2}$ the $(1,-1)$ moiré bands are approximately perpendicular to the original gratings, and their period is given by Eq. (8).

In conclusion, the $T$-convolution theorem allows us to express the moiré profile that we have extracted in the spectral domain in terms of the image domain, too. The importance of the image-domain interpretation of the moiré profile as a $T$ convolution will become clearer in the following.

\section{Moiré Effects in the Superposition of Periodic Dot Screens}

The generalization of the above results into the $2 \mathrm{D}$ case of periodic dot screens is of particular importance for our needs. We therefore summarize below the main results that will be needed later. The full developments can be found in Chap. 4 of Ref. 3.

Let $r_{1}(x, y)$ be a periodic dot screen whose frequencies and orientations are given by the two perpendicular frequency vectors $\mathbf{f}_{1}, \mathbf{f}_{2}$; and let $r_{2}(x, y)$ be a second periodic dot screen whose frequencies and orientations are given by the two perpendicular frequency vectors $\mathbf{f}_{3}, \mathbf{f}_{4}$. The spectrum $R_{1}(u, v)$ of the screen $r_{1}(x, y)$ consists of a $2 \mathrm{D}$ impulse nailbed, whose impulses are located at integer linear combinations of the two fundamental frequency vectors of $r_{1}(x, y), m \mathbf{f}_{1}+n \mathbf{f}_{2}$. Similarly, the spectrum $R_{2}(u, v)$ consists of a $2 \mathrm{D}$ nailbed whose $(m, n)$ th impulse is located at the integer linear combinations $m \mathbf{f}_{3}+n \mathbf{f}_{4}$. The amplitude of the $(m, n)$ th impulse of the $i$ th nailbed is denoted by $a_{m, n}^{(i)}$.

Assume now that we superpose (i.e., multiply) $r_{1}(x, y)$ and $r_{2}(x, y)$. According to the convolution theorem, the spectrum of the superposition is the convolution of the nailbeds $R_{1}(u, v)$ and $R_{2}(u, v)$; this means that a centered copy of one of the nailbeds is placed on top of each impulse of the other nailbed (the amplitude of each copied nailbed being scaled down by the amplitude of the impulse on top of which it has been copied). This convolution gives a "forest" of impulses scattered throughout the
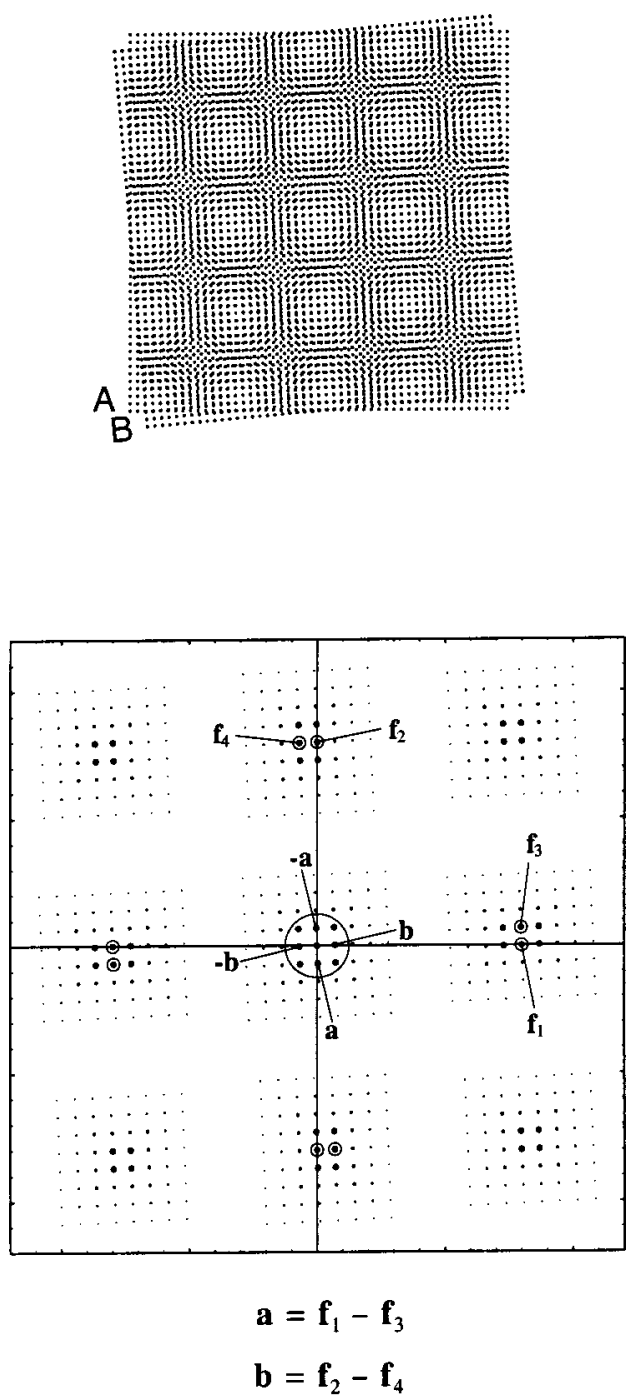

Fig. 6. Superposition of two dot screens with identical frequencies and with an angle difference of $\alpha=5^{\circ}$ (top) and the corresponding spectrum (bottom). Only impulse locations are shown in the spectrum, but not their amplitudes. Encircled points denote the locations of the fundamental impulses of the two original dot screens. Large points represent convolution impulses of the first order (i.e., $\left(k_{1}, k_{2}, k_{3}, k_{4}\right)$ impulses with $k_{i}=1$, 0 , or -1 ); smaller points represent convolution impulses of higher orders. (Note that impulses of only the first few orders are shown; in reality, each impulse cluster extends in all directions ad infinitum.) The circle around the spectrum origin represents the visibility circle. Note that the spectrum origin is closely surrounded by the impulse cluster of the $(1,0,-1,0)$ moiré.

spectrum, as shown in Fig. 6. As we can see in the figure, the spectrum origin is closely surrounded by a full cluster of impulses. The cluster impulses closest to the origin, inside the visibility circle, include the $\left(k_{1}, k_{2}, k_{3}, k_{4}\right)$ impulse, the fundamental impulse of the moiré in question, ${ }^{11}$ and its perpendicular counterpart, the $\left(-k_{2}, k_{1},-k_{4}, k_{3}\right)$ impulse, which is the fundamental impulse of the same moiré in the perpendicular direction. Naturally, each of these two impulses is also accompanied by its respective symmetrical twin to the opposite side of the origin. The locations (frequency vectors) of these four impulses are marked in Fig. 6 by $\mathbf{a},-\mathbf{a}, \mathbf{b}$, and $-\mathbf{b}$. 
If we look attentively at the impulse cluster surrounding the origin, we can see that this cluster is in fact a nailbed whose support is the regular lattice that is spanned by $\mathbf{a}$ and $\mathbf{b}$, the geometric locations of the fundamental moiré impulses $\left(k_{1}, k_{2}, k_{3}, k_{4}\right) \quad$ and $\left(-k_{2}, k_{1},-k_{4}, k_{3}\right)$. This infinite impulse nailbed represents in the spectrum the $2 \mathrm{D}\left(k_{1}, k_{2}, k_{3}, k_{4}\right)$ moiré, and its basis vectors $\mathbf{a}$ and $\mathbf{b}$ determine the period and the two perpendicular directions of the moiré. This impulse nailbed is the $2 \mathrm{D}$ generalization of the moiré comb that we had in the case of line-grating superpositions. Note that the impulse nailbed shown in Fig. 6 belongs to the simplest first-order moiré between two dot screens, the $(1,0,-1,0)$ moiré, which is the $2 \mathrm{D}$ generalization of the $(1,-1)$ moiré between two gratings (Fig. 4).

The full expressions for the location and the amplitude of each of the impulses of the $\left(k_{1}, k_{2}, k_{3}, k_{4}\right)$ moiré nailbed can be found in Ref. 3, Sec. 4.3. Here we will give them only for the $(1,0,-1,0)$ moiré. The location of the $(m, n)$ th impulse in the spectrum is given in this case by

$$
m \mathbf{a}+n \mathbf{b}=m \mathbf{f}_{1}+n \mathbf{f}_{2}-m \mathbf{f}_{3}-n \mathbf{f}_{4} .
$$

For instance, the $(1,0)$ th impulse of the moiré nailbed is the $(1,0,-1,0)$ th impulse of the convolution, and it is located in the spectrum at the point $\mathbf{a}=\mathbf{f}_{1}-\mathbf{f}_{3}$. Similarly, the $(0,1)$ th impulse of this moire nailbed is the $(0,1,0,-1)$ th impulse of the convolution, and it is located in the spectrum at the point $\mathbf{b}=\mathbf{f}_{2}-\mathbf{f}_{4}$.

The amplitude $d_{m, n}$ of the $(m, n)$ th impulse in the $(1,0,-1,0)$ moiré cluster is given by

$$
d_{m, n}=a_{m, n}^{(1)} a_{-m,-n}^{(2)} .
$$

This means that the $(1,0,-1,0)$ moiré-nailbed is simply a term-by-term product of the nailbeds $R_{1}(u, v)$ and $R_{2}(-u,-v)$ of the original screens, that is, where $R_{2}(u, v)$ is inverted (rotated by $180^{\circ}$ ) before the multiplication.

Since we already know the exact locations of the impulses of the moire nailbed, this nailbed can be considered, in fact, to be a product of the two original nailbeds after they have been normalized (rotated and stretched) to fit the impulse locations of the resulting moiré nailbed. Now this moiré nailbed can be extracted from the spectrum convolution. Thus by taking its inverse Fourier transform, we can reconstruct, back in the image domain, the isolated contribution of the moire in question to the image superposition; this is the intensity profile of the moiré, a function in the image domain whose value at each point $(x, y)$ indicates quantitatively the intensity level (more precisely: the reflectance or the transmittance) of the moiré in question. Note that although this moire is visible both in the layer superposition $r_{1}(x, y) r_{2}(x, y)$ and in the extracted moiré intensity profile, the latter does not contain the fine structure of the original layers $r_{1}(x, y)$ and $r_{2}(x, y)$ but only the pure contribution of the extracted moiré itself.

Now, just as in the case of grating superposition (Subsection 2.C), the spectral-domain term-by-term multiplication of the moiré nailbeds as defined by Eq. (15) can be also interpreted, back in the image domain, by using the
2D version of the $T$-convolution theorem (Ref. 3, p. 95). This gives the following result, which is the $2 \mathrm{D}$ generalization of Proposition 2:

Proposition 3. The intensity profile of the $(1,0,-1,0)$ moiré in the superposition of two periodic dot screens with frequencies $\mathbf{f}_{1}, \mathbf{f}_{2}$ and $\mathbf{f}_{3}, \mathbf{f}_{4}$ and an angle difference of $\alpha$ can be seen from the image-domain point of view as the result of a three-stage process:

1. Normalization of the original screens (by linear stretching and rotation transformations) in order to bring both of them to a common period and orientation (that is to make their periods, or their impulse nailbeds in the spectrum, coincide).

2. $T$ convolution of the two normalized screens.

3. Stretching and rotating the resulting normalized moiré intensity profile into its actual scale and orientation, as determined by the vectors $\mathbf{a}=\mathbf{f}_{1}-\mathbf{f}_{3}$ and $\mathbf{b}$ $=\mathbf{f}_{2}-\mathbf{f}_{4}$, or, more explicitly, by Eqs. (5) and (6).

Let us see now how this $T$ convolution sheds a new light on the profile form of the $(1,0,-1,0)$ moiré and explains the striking visual effects observed in superpositions of dot screens such as those in Fig. 3.

\section{Shape of the Moiré Profile}

Case 1. Suppose, first, that one of the superposed screens consists of dots of a given shape (such as the digit 1) and that the other screen consists of tiny white (or transparent) pinholes on a black background. In the $T$ convolution such pinholes play the role of very narrow pulses with amplitude 1 . As shown in Fig. 7(a), the $T$ convolution of such narrow pulses (from one of the screens) and dots of any shape (from the other screen) gives dots of the latter shape, in which the zero values remain at zero, the 1 values are scaled down to the value $A$ (the volume of the narrow white pulse divided by the total period area), and the sharp step transitions are replaced by slightly softer ramps. This means that the dot shape received in the normalized moire period is practically identical to the dot shape of the other screen (in our example, 1) except that its white areas turn darker. However, this normalized moiré period is stretched back into the real size of the moiré period, $T_{M}$, as it is determined by Eqs. (5) and (6). Hence the moiré form in this case is essentially a magnified version of the screen element (the digit 1), where the magnification rate is controlled by the angle $\alpha$ between the two screens.

Case 2. A similar effect, albeit somewhat less impressive, occurs in the superposition where one of the two screens contains tiny black dots [see Figs. 4.4(c) and 4.4(d) of Ref. 3]. Tiny black dots on a white background can be interpreted as inverse pulses of 0 amplitude on a constant background of amplitude 1 . As we can see in Fig. 7(b), the $T$ convolution of such inverse pulses (from one of the screens) and dots of any shape (from the other screen) gives dots of the latter shape, where the zero values are replaced by the value $B$ (the volume under a oneperiod cell of the second screen divided by the period area) and the 1 values are replaced by the value $B-A$ (where $A$ is the volume of the "hole" of the narrow black pulse divided by the period area). This means that the dot shape of the normalized moiré period is similar to the dot shape 

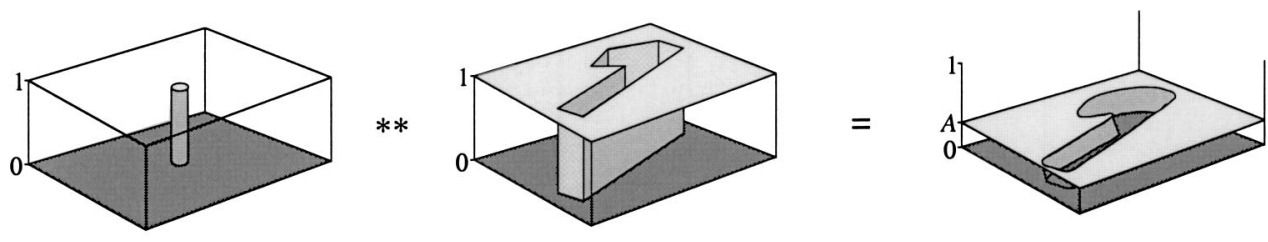

(a)
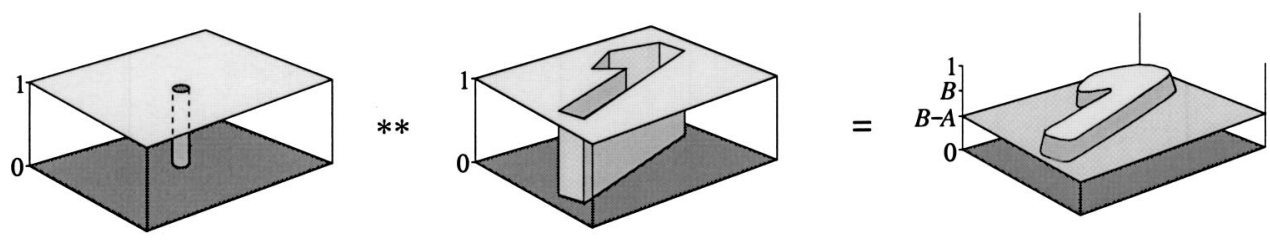

(b)

Fig. 7. (a) Convolution of tiny white dots (from the first screen) with dots of any given shape (from the other screen) gives dots of essentially the same given shape; (b) convolution of tiny black dots (from the first screen) with dots of any given shape (from the other screen) gives dots of essentially the same shape, but in inverse video.

of the second screen except that it appears in inverse video and with slightly softer ramps.

Case 3. When none of the two superposed screens contains tiny dots, either white or black, the profile form of the resulting moire is still a magnified version of the $T$ convolution of the two original screens. As before, this $T$ convolution gives some kind of blending between the two original dot shapes, but this time the resulting shape has a rather blurred or smoothed-out appearance, and the moiré looks less attractive to the eye.

\section{Orientation and Size of the Moiré Cells}

As we can see in Fig. 3, although the $(1,0,-1,0)$ moiré cells inherit the forms of the original screen cells, they do not inherit their orientations. Rather than having the same direction as the cells of the original screens (or an intermediate orientation), the moiré cells appear in a perpendicular direction. This fact is explained as follows.

As we already know, the orientation and the size of the moire are determined by the location of the fundamental impulses of the moire nailbed in the spectrum, i.e., by the location of the basis vectors $\mathbf{a}$ and $\mathbf{b}$. We have seen following Eq. (14) that in the case of the $(1,0,-1,0)$ moiré, these vectors are given by

$$
\begin{aligned}
& \mathbf{a}=\mathbf{f}_{1}-\mathbf{f}_{3}, \\
& \mathbf{b}=\mathbf{f}_{2}-\mathbf{f}_{4} .
\end{aligned}
$$

And indeed, as we can see in Figs. 6 and 8, when the two original screens have the same frequency, these basis vectors are perpendicular to the bisectors of the angles formed between the frequency vectors $\mathbf{f}_{1}, \mathbf{f}_{3}$ and $\mathbf{f}_{2}, \mathbf{f}_{4}$. This means that in this case the $(1,0,-1,0)$ moiré nailbed (and the corresponding moiré profile in the image domain) are closely perpendicular to the original screens $r_{1}(x, y)$ and $r_{2}(x, y)$. The period of this moiré can be found by Eq. (8), which was derived for the $(1,-1)$ moiré between two line gratings with identical periods $T$ and angle difference of $\alpha$.

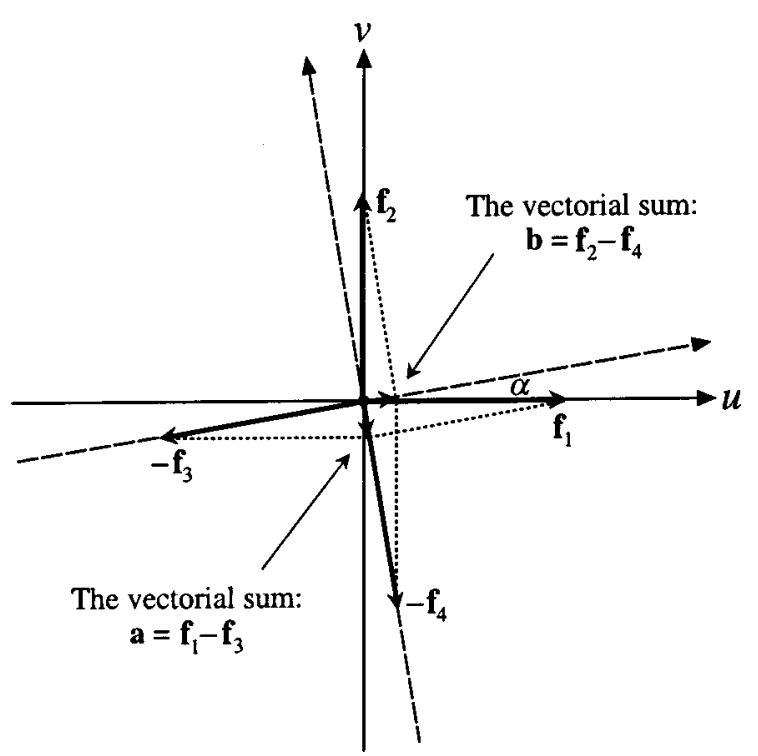

Fig. 8. Detail from Fig. 6 showing the spectral interpretation (vector diagram) of the $(1,0,-1,0)$ moiré between two dot screens with identical frequencies and a small angle difference $\alpha$. The low-frequency vectorial sums $\mathbf{a}$ and $\mathbf{b}$ [which are the geometric locations of the two fundamental impulses of the $(1,0,-1,0)$ moire cluster] are closely perpendicular to the directions of the two original screens: $\mathbf{a}$ is perpendicular to the bisecting direction between $\mathbf{f}_{1}$ and $\mathbf{f}_{3}$, and $\mathbf{b}$ is perpendicular to the bisecting direction between $\mathbf{f}_{2}$ and $\mathbf{f}_{4}$.

\section{GLASS PATTERNS IN THE SUPERPOSITION OF APERIODIC GRATINGS}

Having reviewed the basic concepts of the periodic cases, we are ready now to investigate their aperiodic counterparts. We start our study with the superposition of aperiodic gratings consisting of parallel straight lines (see Fig. 9). This case is simpler and easier to understand than the superposition of aperiodic dot screens, and it will serve as a useful introduction to the case of aperiodic dot screens that we will study in Section 4.

Before we start, let us first introduce a few new terms that will be needed in the following sections. 
Definition 1. Two layers (line gratings, dot screens, etc.) will be called isometric if the individual elements in the two layers (lines, dots, etc.) have the same locations.

For example, if dot screen $r_{1}(x, y)$ consists of arbitrarily located circular dots, and dot screen $r_{2}(x, y)$ is obtained by replacing each of the circular dots of $r_{1}(x, y)$ by a triangular dot that is centered at the same location, then $r_{1}(x, y)$ and $r_{2}(x, y)$ are isometric. Note that the size of the triangular dots need not be identical to the size of the original circular dots; all that we require is that they be centered at the same locations.

Definition 2. Two layers (line gratings, dot screens, etc.) will be called congruent if they can be made isometric (that is, brought into coincidence, element on element) by rotations, translations, or any combination thereof.

For example, any two periodic layers having the same periodicity (or the same frequencies) are congruent.

Definition 3. Two layers (line gratings, dot screens, etc.) will be called similar if they can be made congruent by a linear spatial scaling. (Note that by spatial scaling we mean a spatial expansion or contraction, not a scaling in the function's amplitude.)

It follows, therefore, that similar layers can be brought to coincidence, element on element, by rotations, translations, spatial scalings, or any combination thereof. Congruent layers, however, have the same spatial scaling, and they can have only different rotations or translations.

For example, any two periodic line gratings with periods $T_{1}=T_{2}$ are congruent; but if their periods $T_{1}$ and $T_{2}$ are different, they are no longer congruent but only similar.

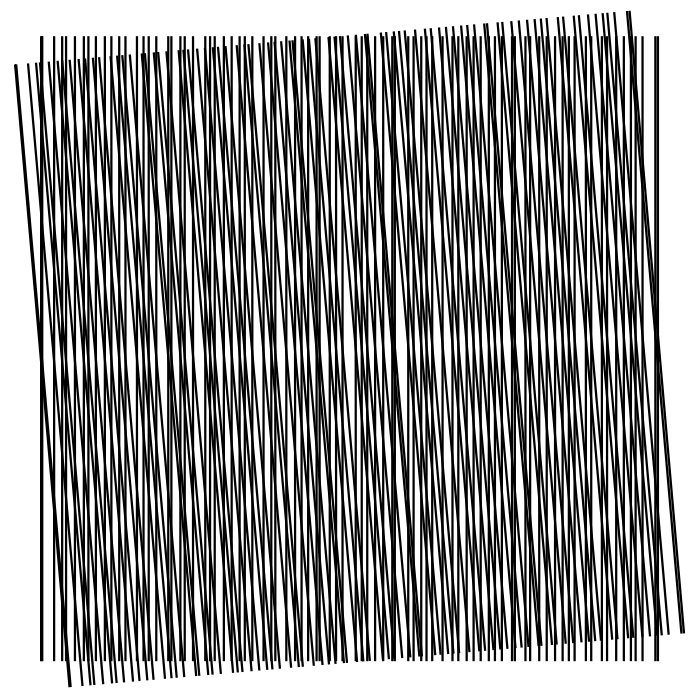

(a)
Note that we will generally be interested in congruent or similar layers that differ from each other only by slight rotation or spatial-scaling transformations so that a moiré effect (or Glass pattern) becomes visible in their superposition.

Finally, some cases of particular interest are introduced by the following definition:

Definition 4. An aperiodic layer with fixed element shapes is a layer that is composed of identical elements whose shapes and profiles are fixed but whose locations are arbitrary (random, pseudorandom, or deterministic). In particular, an aperiodic dot screen is said to have a fixed $d o t$ shape if it is composed of dots whose shapes and profiles are identical while their locations are arbitrary (random, pseudorandom, or deterministic). Such screens can be obtained, for example, by randomizing the dot locations of an initially periodic dot screen. An aperiodic line grating is said to have a fixed line shape if it consists of parallel lines having a fixed intensity profile but varying distances; the line distances may be random, pseudorandom, or deterministic.

\section{A. Superposition of Correlated Gratings}

Suppose we are given two aperiodic gratings $r_{1}(x, y)$ and $r_{2}(x, y)$ as shown in Figs. 10(a) and 10(b). We assume, first, that the two gratings $r_{1}(x, y)$ and $r_{2}(x, y)$ are congruent and that only their orientations are slightly different. As shown in Figs. 9(a) and 10(c), such aperiodic gratings give a clearly visible linear Glass pattern in their superposition. Because each of the original gratings is constant along its lines, i.e., perpendicularly to its main direction, its spectrum $R_{i}(u, v)$ consists of a line impulse (a "blade") passing through the origin that is oriented along the grating's main direction [see Figs. 10(d) and 10(e)]. A line impulse is a generalized function that runs along a $1 \mathrm{D}$ line through the plane and is null everywhere

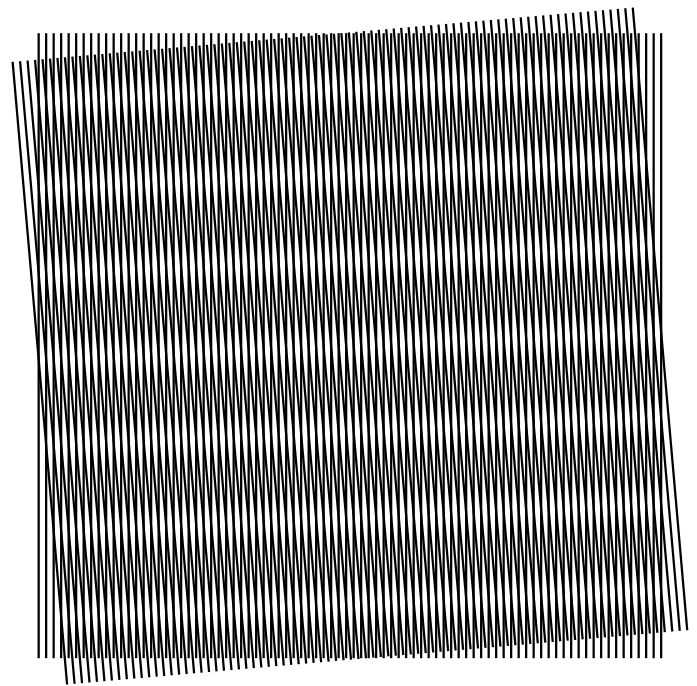

(b)

Fig. 9. (a) Superposition of two identical aperiodic line gratings with a small angle difference gives a moiré effect in the form of a linear Glass pattern passing through the center of rotation. (b) When the superposed layers are periodic, a linear Glass pattern is still generated through the center of rotation, but owing to the periodicity of the layers, this pattern is periodically repeated throughout the superposition, thus generating a periodic moiré pattern. 


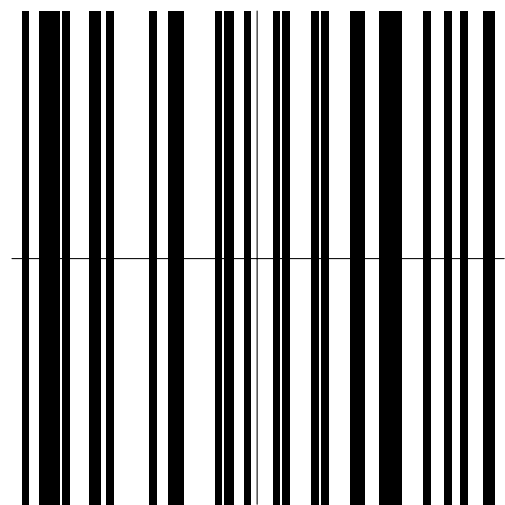

(a)

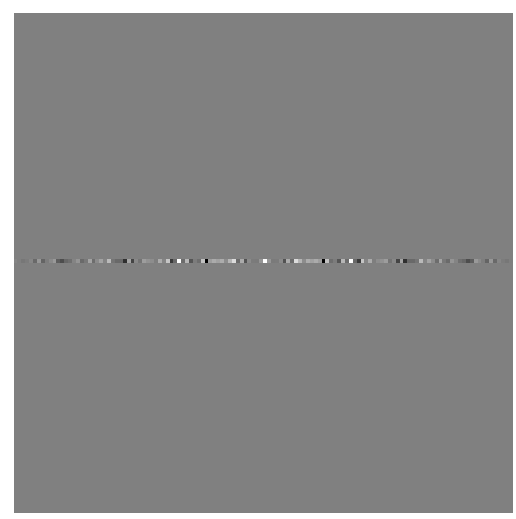

(d)

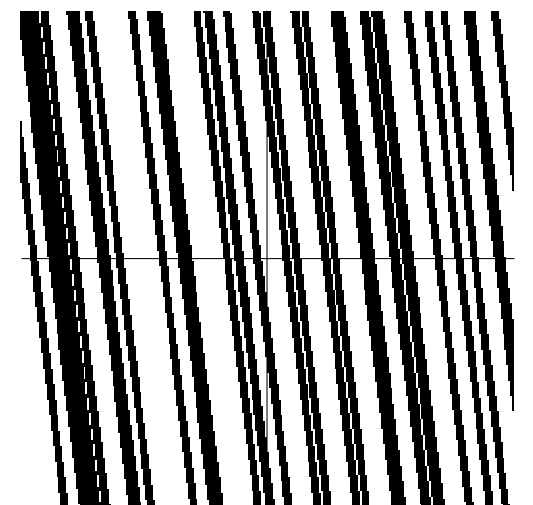

(b)

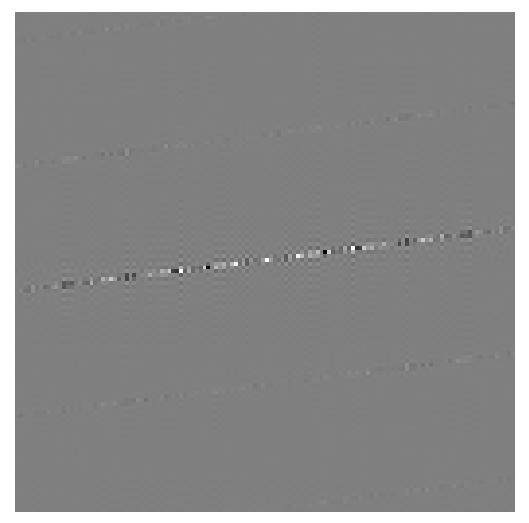

(e)

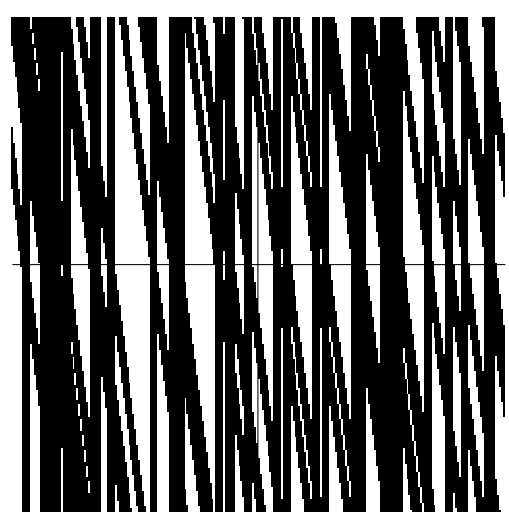

(c)

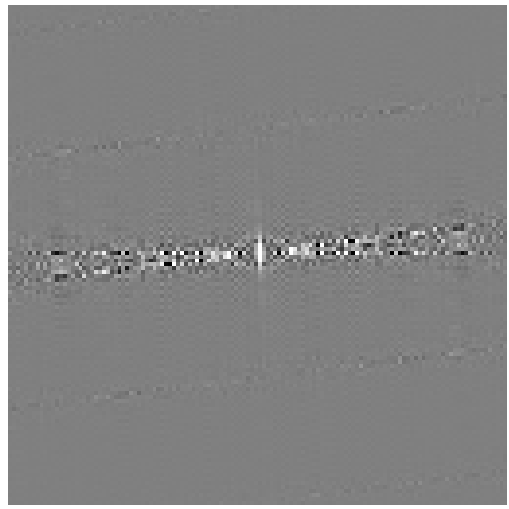

(f)

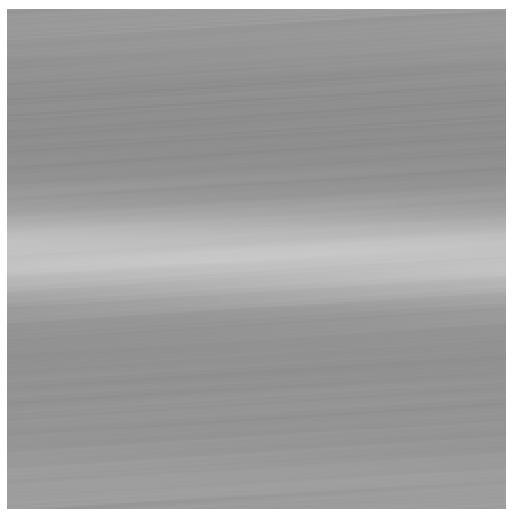

(g)

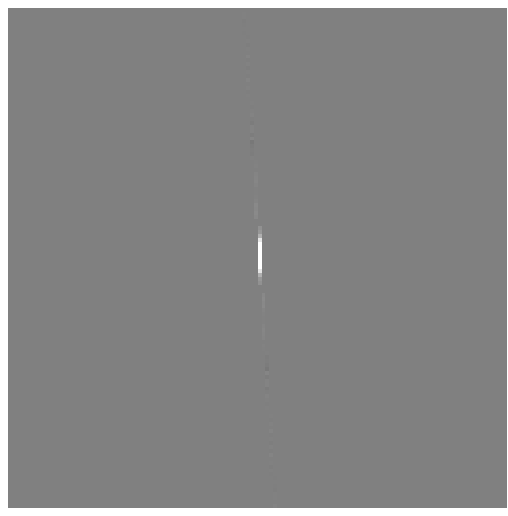

(h)

Fig. 10. Correlated aperiodic line gratings (a) and (b) and their superposition (c) in the image domain; their respective spectra [obtained by 2D fast Fourier transform (FFT)] are the infinite Hermitian blades (d) and (e) and their convolution (f). The white perpendicular line in (f) is the infinite Hermitian blade that represents the Glass pattern seen in (c). This Hermitian blade is shown isolated in (h), after its extraction from the spectrum convolution (f). The amplitude at each point of this blade is the point-by-point product of the respective amplitudes of the blades (d) and (e) taken head to tail. In (g) is shown the image-domain function that corresponds to the spectrum (h), as obtained by inverse FFT of $(\mathrm{h})$; this is the intensity profile of the Glass pattern shown in (c). Note that unlike its periodic counterpart (Fig. 4 ), the present figure has been obtained by digital simulation; the scaling and angle parameters used here are slightly different in order to reduce aliasing and other FFT artifacts. Only the real parts of the spectra are shown; the complex amplitudes of the blades (d) and (e) are similar to those shown in Fig. 11. 
else. A line impulse can be graphically illustrated as a blade whose behavior is continuous (or diffuse; see end of Subsection 2.B) along its 1D line support but impulsive in the perpendicular direction.

As our aperiodic gratings in the image domain are real valued but are not necessarily symmetric about the origin, it follows that their spectra are Hermitian (Ref. 8, p. 15). This means that the amplitude of each of the blades in Figs. 10(d) and 10(e) is complex valued, where the real part is symmetric with respect to the origin and the imaginary part is antisymmetric (see Fig. 11).

Consider now the superposition of our aperiodic gratings $r_{1}(x, y)$ and $r_{2}(x, y)$ [Fig. 10(c)]. Since this superposition is the product of the two original gratings, it follows according to the convolution theorem that the spectrum of the superposition is the convolution of the original spectra. The convolution of two $2 \mathrm{D}$ functions can be illustrated graphically by the "move-and-multiply" method (see, for example, Ref. 12 pp. 13 and 14 or Ref. 13 pp. 291 and 292): We first rotate one of the original functions by $180^{\circ}$, and then we determine the value of the convolution at any point $(u, v)$ in the plane as the volume under the product of the two functions when the origin of the moving function is located at the point $(u, v)$. In our case, the convolution is performed between two blades $R_{1}(u, v)$ and $R_{2}(u, v)$ that have different orientations, so the value of the convolution at any point in the $(u, v)$ plane is simply the product of the two blades $R_{1}(u, v)$ and $R_{2}(-u,-v)$ at their intersection point. It follows, therefore, that unlike in the periodic case, the spectrum of

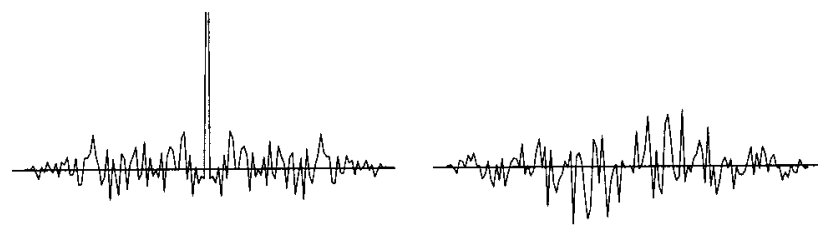

(a)

(b)

Fig. 11. Amplitude of the Hermitian blade of Fig. 10(d). (a) Real part of the amplitude, (b) the imaginary part of the amplitude. the grating superposition [i.e., the convolution of the two line impulses; see Fig. 10(f)] is no longer impulsive but rather a $2 \mathrm{D}$ continuous (or diffuse) function. We will call this function, for the sake of our discussion, a "hump." This hump is Hermitian, since it is the spectrum of the grating product $r_{1}(x, y) r_{2}(x, y)$, which is obviously real valued.

Consider now the cross section (infinitely thin slice) of this hump that passes through the spectrum origin perpendicularly to the line bisecting the original line spectra of Figs. 10(d) and 10(e). This section, which appears in Fig. 10(f) as a white line, can be extracted by setting all the rest of the spectrum convolution to zero. Clearly, this isolated section [see Fig. 10(h)] is a line impulse; moreover, the amplitude of this line impulse is, by construction, a spatially scaled version of the product of the two original line impulses, one of which has been inverted (rotated by $180^{\circ}$ ) before the multiplication. Thus, if we consider in the spectral domain each of the two original line impulses $P_{1}$ and $P_{2}$ as well as our new line impulse $P$ as a $1 D$ function running along its own main direction, we obtain

$$
P\left(f_{M}\right)=P_{1}(f) P_{2}(-f),
$$

where $f_{M}=c f, c$ being a scaling factor that depends on the angle difference $\alpha$ between the two original line impulses (i.e., the angle between the original gratings). The value of $f_{M}$ for any given point $f$ along the original blades can be found as shown in Fig. 12:

$$
\sin (\alpha / 2)=\frac{f_{M} / 2}{f} ;
$$

hence

$$
\begin{aligned}
f_{M} & =2 f \sin (\alpha / 2), \\
c & =f_{M} / f=2 \sin (\alpha / 2) .
\end{aligned}
$$

Note that the functions $P_{1}(f)$ and $P_{2}(f)$ are identical to the spectra of the original layers, $R_{1}(u, v)$ and $R_{2}(u, v)$ except that they are expressed in terms of different basis vectors.

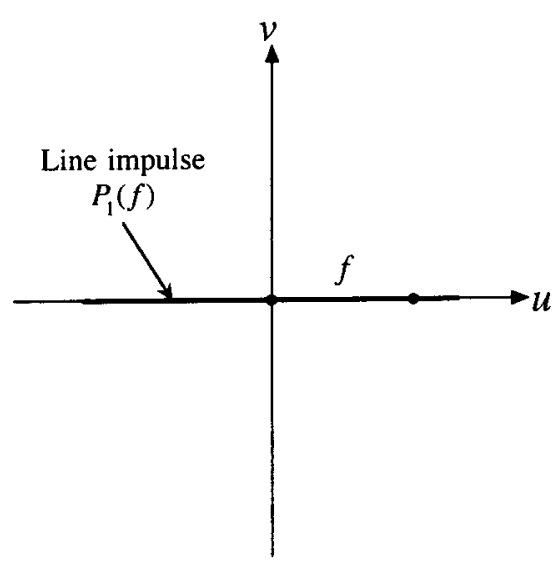

(a)

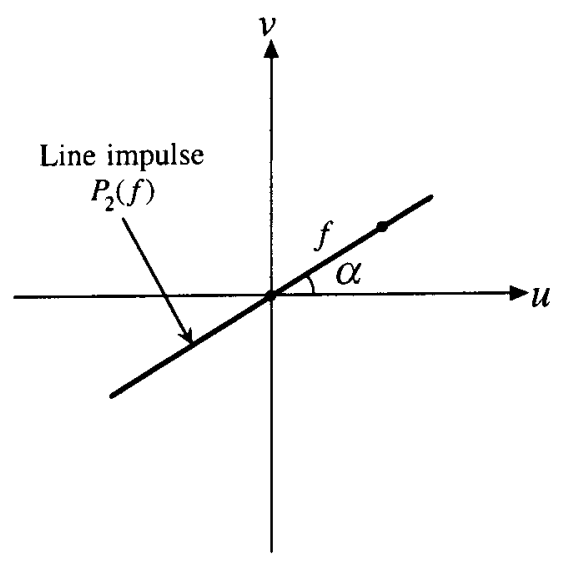

(b)

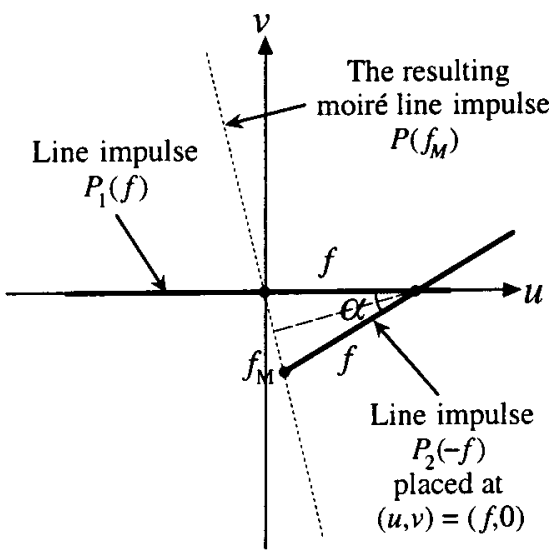

(c)

Fig. 12. Geometric consideration in the frequency domain illustrating the scaling ratio between the original line spectra shown in (a) and (b) and the isolated line spectrum shown as a dotted line in (c). 
We immediately recognize that Eq. (17) is, in fact, a generalization of Eq. (13) of the $(1,-1)$ moiré between two periodic gratings: It generalizes the purely impulsive spectra of periodic gratings into the continuous (or diffuse) line-impulse spectra of aperiodic gratings. Furthermore, Eq. (18) is identical to Eq. (7), which was obtained in the case of the $(1,-1)$ moire between two periodic gratings with the same frequency $\left(f_{1}=f_{2}\right)$; the only difference is that in our case we cannot proceed from Eq. (18) to the language of periods, as we did in the periodic case [Eq. (8)], because in an aperiodic case there is no equivalent to the relation $T=1 / f$.

It should be noted that Eqs. (17) and (18) above were derived for the case in which the original gratings have undergone only rotations but not scalings. (Recall our assumption in the beginning of Subsection 3.A that our two aperiodic gratings are congruent and only their orientations are slightly different.) If, in addition to the rotation, each of the gratings undergoes a scaling transformation, Eqs. (17) and (18) become

$$
P\left(f_{M}\right)=P_{1}\left(f_{1}\right) P_{2}\left(-f_{2}\right)
$$

and

$$
f_{M}=\left(f_{1}{ }^{2}-2 f_{1} f_{2} \cos \alpha+f_{2}{ }^{2}\right)^{1 / 2} .
$$

These are generalizations into the aperiodic case of Eqs. (13) and (9) of the (1, -1) moiré between two periodic gratings with different frequencies $f_{1} \neq f_{2}$. Note that in this case the cross section [Eq. (20)] through the spectrum convolution is no longer oriented perpendicularly to the bisector of the original line impulses; it can be shown that its orientation is given, just like in the periodic case [see Eq. (11)], by

$$
\varphi_{M}=\arctan \left(\frac{T_{2} \sin \theta_{1}-T_{1} \sin \theta_{2}}{T_{2} \cos \theta_{1}-T_{1} \cos \theta_{2}}\right) .
$$

We see, therefore, that Eqs. (17) and (20) are the aperiodic counterparts of Eq. (13) that we have obtained in Subsection 2.C in the case of the $(1,-1)$ moiré between periodic gratings. The $(1,-1)$ moiré is indeed the simplest and most common type of a $\left(k_{1}, k_{2}\right)$ moiré between two periodic gratings; the reason that this particular moire is the basis for the generalization into the aperiodic case will be explained later, in Section 5 .

Hence, interestingly, the extension of the periodic case into the aperiodic case is most naturally done in the spectral domain, where, instead of considering three impulse combs with discrete frequencies $n \mathbf{f}_{1}, n \mathbf{f}_{2}$, and $n \mathbf{f}_{M}$ $=n\left(\mathbf{f}_{1}-\mathbf{f}_{2}\right), n \in \mathbb{Z}$, as we did in the periodic case, we consider three line impulses with continuous frequencies $n \mathbf{f}_{1}, n \mathbf{f}_{2}$, and $n \mathbf{f}_{M}=n\left(\mathbf{f}_{1}-\mathbf{f}_{2}\right)$, where $n \in \mathbb{R}$. Each of these line impulses is therefore a continuous extension of the corresponding impulse comb, where the gaps between the discrete impulse locations have been filled in. In this continuous case, the basis vectors $\mathbf{f}_{1}, \mathbf{f}_{2}$, and $\mathbf{f}_{M}$ along the respective line impulses simply indicate the corresponding unit frequency rather than a discrete fundamental frequency that determines a periodicity in the image domain.

The relationship between the discrete spectra of the periodic case and the continuous spectra of the aperiodic case can be shown even better by rewriting Eq. (20) in an equivalent form, using the basis vectors $\mathbf{f}_{M}, \mathbf{f}_{1}$, and $\mathbf{f}_{2}$ :

$$
P\left(n \mathbf{f}_{M}\right)=P_{1}\left(n \mathbf{f}_{1}\right) P_{2}\left(-n \mathbf{f}_{2}\right), \quad n \in \mathbb{R},
$$

where $f_{1}, f_{2}$, and $f_{M}$ of Eq. (20) are the lengths of the vectors $n \mathbf{f}_{1}, n \mathbf{f}_{2}$, and $n \mathbf{f}_{M}$. This can be written in a more concise form, where the coordinate $n$ of each of the line impulses is expressed in terms of its own basis vector, $\mathbf{f}_{M}$, $\mathbf{f}_{1}$, or $\mathbf{f}_{2}$ :

$$
P(n)=P_{1}(n) P_{2}(-n), \quad n \in \mathbb{R} .
$$

This is clearly the continuous counterpart of Eq. (13). Note that in Eq. (13), too, the index $n$ in each of the three components refers to the $n$th impulse of a different comb, and the corresponding impulse location in the $u, v$ plane is determined in terms of the fundamental frequency vector of its own comb: $\mathbf{f}_{M}, \mathbf{f}_{1}$, or $\mathbf{f}_{2}$.

This relationship between the periodic and the aperiodic cases can be graphically illustrated by comparing the spectra of Fig. 4 (the periodic case) and Fig. 10 (its aperiodic counterpart).

Extending this relationship between Fig. 4 and Fig. 10 one step further, we may guess that the line impulse of Fig. 10(h), which we have extracted from the spectrum convolution Fig. 10(f), is the spectrum of the moiré effect that is generated in the superposition of the two gratings, namely, in our case, the linear Glass pattern that is clearly visible in Fig. 10(c). And, indeed, if we apply an inverse Fourier transform on the extracted line impulse of Fig. 10(h), we obtain back in the image domain [see Fig. $10(\mathrm{~g})$ ] the intensity profile of the isolated Glass pattern of Fig. 10(c). Note that just as in the periodic case (Fig. 4), this extracted Glass pattern no longer contains the fine structure of the original layers but only the pure contribution of the Glass pattern itself.

However, since the extracted line impulse of Fig. 10(h) is a normalized product of the line impulses of the original spectra, as stated by Eq. (20), it follows from the convolution theorem that the extracted intensity profile of the Glass pattern, shown in Fig. 10(g), is simply a normalized convolution of the intensity profiles of the two original gratings.

This result can therefore be formulated as an extension of Proposition 2 of Subsection 2.C into the aperiodic case:

Proposition 4. The intensity profile of the linear Glass pattern that is generated in the superposition of two similar aperiodic line gratings (namely, isometric gratings that have undergone linear rotation and spatial-scaling transformations) can be seen from the image-domain point of view as the result of a three-stage process:

1. Normalization of each of the original gratings by applying on it the inverse of its rotation and spatialscaling transformation.

2. Convolution of the two normalized gratings, giving the normalized linear Glass pattern.

3. Rotating and spatially scaling the normalized Glass pattern into its actual scale and orientation, as determined by Eqs. (21) and (22).

This result can be stated more formally as the aperiodic counterpart of Proposition 10.2 of Ref. 3, p. 330: 
Proposition 5. Let $r_{1}(x, y)$ and $r_{2}(x, y)$ be two aperiodic line gratings that are obtained by applying linear transformations (scalings, rotations, etc.) $g_{1}(x, y)$ and $g_{2}(x, y)$, respectively, on two isometric aperiodic line gratings that have the intensity profiles $p_{1}\left(x^{\prime}\right)$ and $p_{2}\left(x^{\prime}\right)$

$$
r_{1}(x, y)=p_{1}\left(g_{1}(x, y)\right), \quad r_{2}(x, y)=p_{2}\left(g_{2}(x, y)\right) .
$$

Then the Glass pattern $m(x, y)$ in the superposition of $r_{1}(x, y)$ and $r_{2}(x, y)$ is given by

$$
m(x, y)=p(g(x, y))
$$

where

1. $p\left(x^{\prime}\right)$, the normalized intensity profile of the Glass pattern, is the convolution of the normalized intensity profiles of the original gratings,

$$
p\left(x^{\prime}\right)=p_{1}\left(x^{\prime}\right) * p_{2}\left(-x^{\prime}\right) ;
$$

2. $g(x, y)$, the linear transformation that brings $p\left(x^{\prime}\right)$ back into the actual scale and orientation of the Glass pattern $m(x, y)$ is given by

$$
g(x, y)=g_{1}(x, y)-g_{2}(x, y) .
$$

Note that if the explicit expressions of the linear transformations $g_{1}(x, y)$ and $g_{2}(x, y)$ are given by

$$
\begin{aligned}
& g_{1}(x, y)=u_{1} x+v_{1} y=\mathbf{f}_{1} \cdot \mathbf{x} \\
& \quad \text { with } \mathbf{f}_{1}=\left(u_{1}, v_{1}\right), \quad \mathbf{x}=(x, y), \\
& g_{2}(x, y)=u_{2} x+v_{2} y=\mathbf{f}_{2} \cdot \mathbf{x} \\
& \text { with } \mathbf{f}_{2}=\left(u_{2}, v_{2}\right), \quad \mathbf{x}=(x, y) ;
\end{aligned}
$$

then we have

$$
\begin{aligned}
g(x, y)=\left(u_{1}-u_{2}\right) x+\left(v_{1}-v_{2}\right) y=\mathbf{f}_{M} \cdot \mathbf{x} \\
\text { with } \mathbf{f}_{M}=\mathbf{f}_{1}-\mathbf{f}_{2}, \quad \mathbf{x}=(x, y) .
\end{aligned}
$$

This general formulation embraces formulas (21) and (22) and their particular case, Eq. (18), which simply give explicit expressions for the length and the orientation of the vector $\mathbf{f}_{M}=\mathbf{f}_{1}-\mathbf{f}_{2}$. But whereas in the periodic case $\mathbf{f}_{1}, \mathbf{f}_{2}$ and $\mathbf{f}_{M}=\mathbf{f}_{1}-\mathbf{f}_{2}$ were the frequency vectors of the original periodic gratings and of the resulting moiré, here they are simply the basis vectors of the respective spectra, and they convey only the scaling and the orientation of the aperiodic gratings-but not any notion of periodicity. $^{14}$

Thus, by extending our moiré theory from the periodic case to the aperiodic case, we have succeeded in extracting the isolated Glass pattern from the grating superposition both in the spectral domain and in the image domain. The full significance of these results will be better appreciated in Section 4, when we will discuss their 2D counterparts.

Remark 1. Note that since the intensity profiles $p_{i}($ ) are not periodic, we are dealing here with convolution and not, as in Section 2, with $T$ convolution (the periodic counterpart of convolution). Whereas this convolution gives a single Glass pattern, $T$ convolution gives in the periodic case infinitely many moiré replicas. The reason is that in the periodic case, each time the moving layer in the move- and-multiply convolution process advances by a full period, the same values are recorded in the result on a periodic basis.

\section{B. Superposition of Uncorrelated Gratings}

What would have happened now if the original gratings $r_{1}(x, y)$ and $r_{2}(x, y)$ were not isometric (or at least correlated) before undergoing the linear rotation and scaling transformations $g_{1}(x, y)$ and $g_{2}(x, y)$ ? As shown in Fig. 13, two uncorrelated gratings (a) and (b) do not generate a Glass pattern in their superposition (c). Let us see how we can explain this fact using the theory we have developed above.

First of all, we note that the convolution in Eq. (25) is, in fact, the cross correlation of $p_{1}\left(x^{\prime}\right)$ and $p_{2}\left(x^{\prime}\right)$ (see Ref. 13, p. 172):

$$
p_{1}\left(x^{\prime}\right) \star p_{2}\left(x^{\prime}\right)=p_{1}\left(x^{\prime}\right) * p_{2}\left(-x^{\prime}\right) .
$$

Intuitively, the cross correlation of two functions is obtained by a move-and-multiply process similar to that of convolution, except that none of the two original functions is inverted (or rotated by $180^{\circ}$ ) before the operation. Suppose, first, that our two original layers are isometric. Therefore when $p_{1}\left(x^{\prime}\right)$ and $p_{2}\left(x^{\prime}\right)$ are located, during the move-and-multiply process, in full or almost full coincidence, the resulting cross-correlation values (the volume under the product function) are high; but when the two layers are out of coincidence, the resulting values fluctuate arbitrarily around some mean value. This results in much higher values around the center of the cross correlation [see Fig. 14(a)]. And indeed, this area of higher values in the center of the cross correlation simply represents the brighter zone in the center of the intensity profile of the Glass pattern [see Fig. 10(g)]. A similar result will be obtained whenever the two original layers are well correlated, giving a "privileged" area of brighter values in the center, which corresponds to the Glass pattern. But when the original layers are not correlated, there exists no mutual locus where all of their elements fall on top of one another, and therefore their cross correlation does not contain such a privileged area; it simply fluctuates around the same mean level throughout [see Fig. 14(b)]. Hence no visible structure (Glass pattern) appears in the superposition. Note, however, that the terms "high values" and "brighter" that we have used above to describe the privileged area in the center of the cross correlation are not always appropriate. This will be explained in Remark 2 .

Remark 2. It should be noted that the privileged area in the center of the cross correlation of two correlated layers is not necessarily brighter. If the two layers involved are correlated and each of them has fixed element shapes, it follows that when the layers are in full or almost full coincidence during the move-and-multiply process, all of their individual elements coincide simultaneously in the same manner. Therefore in this privileged area, the values of the full-layer convolution (or cross correlation) are determined basically by the convolution (or cross correlation) of a single element from each layer. But although in many cases this indeed gives high values in the privileged area, in other circumstances the result may have a 


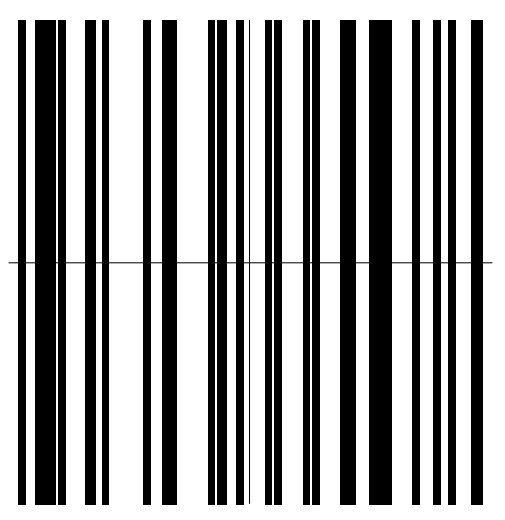

(a)

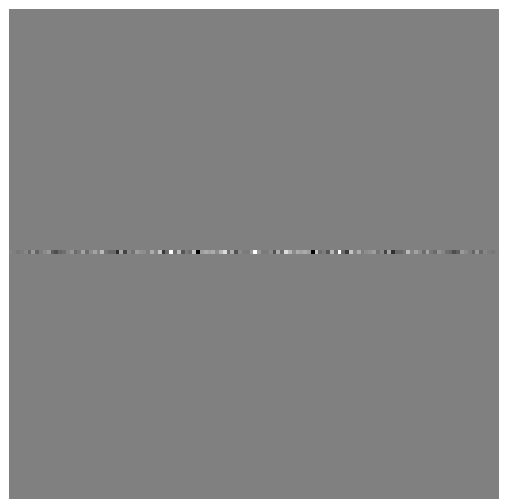

(d)

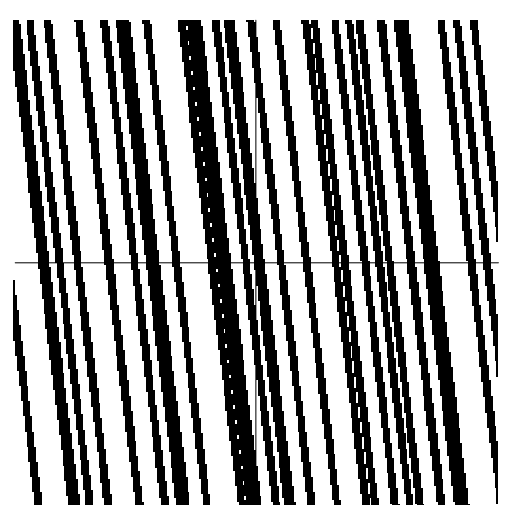

(b)

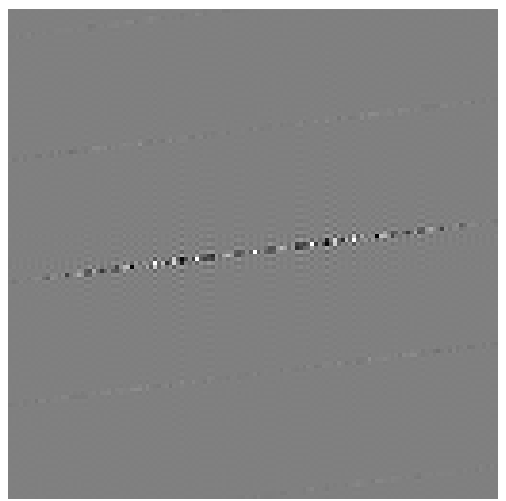

(e)

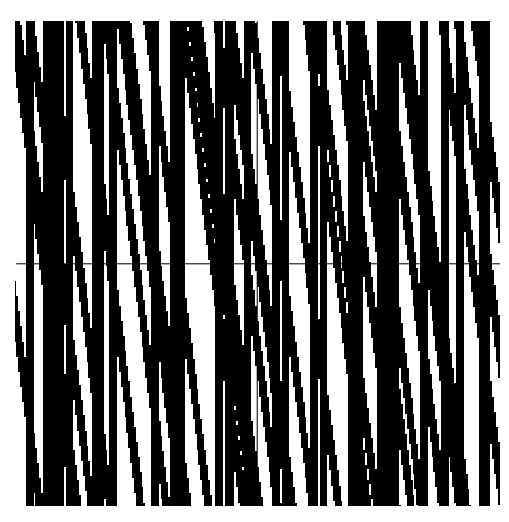

(c)

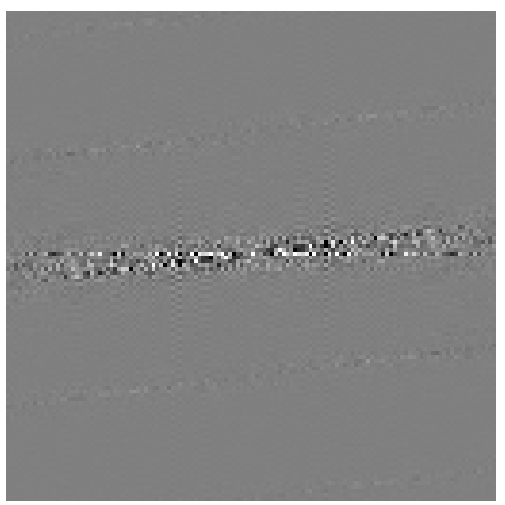

(f)

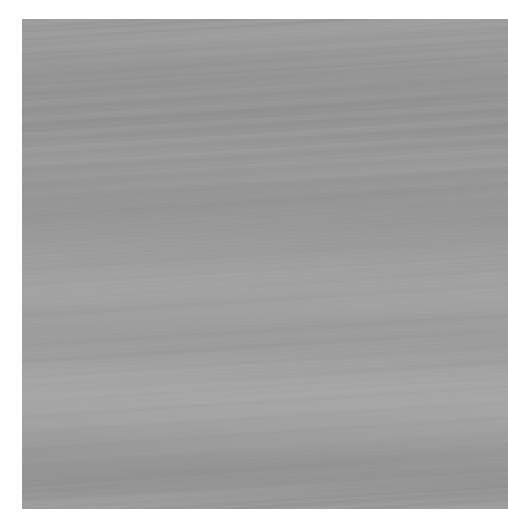

(g)

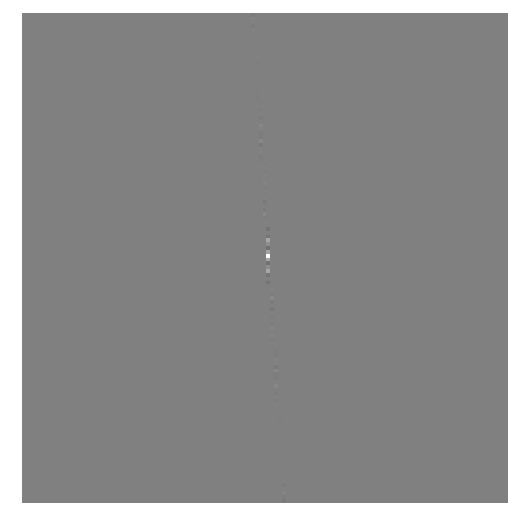

(h)

Fig. 13. Same as in Fig. 10, but with two different aperiodic line gratings (a) and (b). No Glass pattern is generated in their superposition (c). 


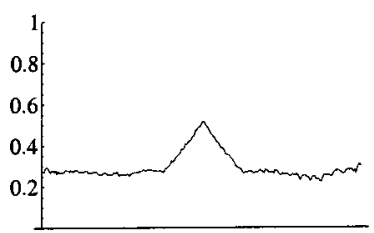

(a)

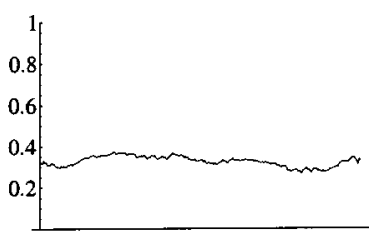

(b)
Fig. 14. (a) Cross section through the extracted Glass pattern of Fig. 10(g), showing its intensity profile. (b) Cross section through Fig. 13(g). Clearly, when the original gratings are not correlated, no Glass pattern is visible in their superposition nor in the extracted intensity profile.

more interesting shape. In Subsection 4.B this will be illustrated in the 2D case through Fig. 7.

In conclusion, it has been shown that the fundamental relationship between the image domain and the spectral domain holds also in the generalized case in which the superposed gratings are no longer periodic. This is illustrated clearly by Figs. 4 and 10. The main differences between periodic and aperiodic cases are that in aperiodic cases our spectra consist of continuous structures instead of discrete impulses and that frequency considerations in the spectral domain, such as those leading to Eq. (18) (see Fig. 12), can no longer be interpreted in the image domain in terms of periods.

\section{GLASS PATTERNS IN THE SUPERPOSITION OF APERIODIC DOT SCREENS}

Having understood the simpler case of aperiodic line gratings, we are ready now to proceed to the superposition of aperiodic dot screens.

\section{A. Superposition of Correlated Screens}

Suppose we are given two aperiodic dot screens $r_{1}(x, y)$ and $r_{2}(x, y)$. We assume, first, that the two screens are either congruent or similar and that only their orientations (and possibly their spatial scalings) are slightly different. As shown in Fig. 1(a), such aperiodic dot screens give a clearly visible Glass pattern in their superposition.

Unlike in the case of aperiodic gratings, the spectrum of an aperiodic screen is no longer a line impulse but a $2 \mathrm{D}$ continuous (or diffuse) hump, which is, of course, Hermitian, since our aperiodic screen is real valued.

Consider now the superposition of our aperiodic screens $r_{1}(x, y)$ and $r_{2}(x, y)$. Since this superposition is the product of the two original gratings, it follows according to the convolution theorem that the spectrum of the superposition is the convolution of the original spectra. Hence, unlike in the periodic case, this spectrum convolution is not impulsive but rather a $2 \mathrm{D}$ continuous (or diffuse) hump.

Following our experience from the previous cases, and as an extension of Fig. 4(h) and Fig. 10(h), we would like now to extract from this convolution the $2 \mathrm{D}$ spectrum that belongs to the isolated Glass pattern. Let us try, therefore, to identify the spectrum that we would like to extract. As we did in the case of aperiodic gratings (Section 3 ), we consider the simplest moiré between two periodic screens, namely, the $(1,0,-1,0)$ moiré. The reason that this particular moiré is the only one that can be generalized into the aperiodic case will be explained later, in Section 5. As we already know, the impulse amplitudes of the $(1,0,-1,0)$ moiré between periodic screens are given by Eq. (15):

$$
d_{m, n}=a_{m, n}^{(1)} a_{-m,-n}^{(2)} \quad \text { with } m, n \in \mathbb{Z} .
$$

Note that in this equation the indices $m, n$ in each of the three components refer to the $m, n$th impulse of a different nailbed. The location of this impulse in the $u, v$ plane is determined in terms of the fundamental frequency vectors of its own nailbed, which are, respectively, $\mathbf{a}=\mathbf{f}_{1}-\mathbf{f}_{3}$ and $\mathbf{b}=\mathbf{f}_{2}-\mathbf{f}_{4}, \mathbf{f}_{1}$ and $\mathbf{f}_{2}$, and $\mathbf{f}_{3}$ and $\mathbf{f}_{4}$ (see Fig. 6).

Now, being inspired by what we did in the case of aperiodic gratings [Eq. (24)], we consider the continuous extension of the spectrum given by Eq. (15), namely,

$$
P(m, n)=P_{1}(m, n) P_{2}(-m,-n) \quad \text { with } m, n \in \mathbb{R},
$$

where the coordinates $m, n$ of each of the three functions is expressed in terms of its own basis vectors. Note that $P_{1}(m, n)$ and $P_{2}(m, n)$ are identical to the spectra of the original screens, $R_{1}(u, v)$ and $R_{2}(u, v)$ except that they are expressed in terms of different basis vectors, as explained below.

As we remember, we have assumed that our superposed screens are congruent (or similar). This means that the original screens were initially isometric, but before being superposed, each of them was linearly transformed (scaled and rotated). Thus the coordinates $m, n$ in $P_{1}(m, n)$ are expressed in terms of the basis vectors $\mathbf{f}_{1}, \mathbf{f}_{2}$ of the first spectrum after it has been scaled and rotated; and similarly, the coordinates $m, n$ in $P_{2}(m, n)$ are expressed in terms of the basis vectors $\mathbf{f}_{3}, \mathbf{f}_{4}$ of the second spectrum after it has been scaled and rotated. The resulting spectrum, $P(m, n)$, is defined as the product of $P_{1}(m, n)$ and $P_{2}(-m,-n)$, but its coordinates $m, n$ are expressed in terms of the basis vectors $\mathbf{a}=\mathbf{f}_{1}-\mathbf{f}_{3}$ and $\mathbf{b}=\mathbf{f}_{2}-\mathbf{f}_{4}$.

Therefore Eq. (27) can be written, back in our usual $u, v$ coordinate system, as follows:

$$
P(m \mathbf{a}+n \mathbf{b})=P_{1}\left(m \mathbf{f}_{1}+n \mathbf{f}_{2}\right) P_{2}\left(-m \mathbf{f}_{3}-n \mathbf{f}_{4}\right) .
$$

Now, as we did in the previous cases, we wish to extract $P(m, n)$ from the spectrum convolution. However, in the present case this seems to be rather hopeless, because unlike in the cases we have studied previously, both the full convolution and the spectrum we wish to extract from it are $2 \mathrm{D}$ humps that overlap each other and are not separable. In other words, $P(m, n)$ cannot be separated or isolated by setting the rest of the spectrum convolution to zero, as was the case in the previous sections (see Figs. 4, 6, and 10). Nevertheless, we can still synthesize this isolated spectrum, using Eq. (27). And indeed, if we apply an inverse Fourier transform on the extracted hump $P(m, n)$, we obtain, back in the image domain, the intensity profile of the isolated Glass pattern. Note that just like in the periodic case, this extracted Glass pattern does not contain the fine structure of the original layers but only the pure contribution of the Glass pattern itself. 
But since the extracted function $P(m, n)$ is a normalized product of the spectra of the original screens, as stated by Eq. (27), it follows from the convolution theorem that the extracted intensity profile of the Glass pattern is simply a normalized convolution of the intensity profiles of the two original screens. This result can therefore be formulated as an extension of Proposition 3 (Subsection 2.D) into the aperiodic case.

Proposition 6. The intensity profile of the Glass pattern that is generated in the superposition of two similar aperiodic dot screens (i.e., isometric screens that have undergone linear rotation and spatial-scaling transformations) can be seen from the image-domain point of view as the result of a three-stage process:

1. Normalization of each of the original screens by applying on it the inverse of its rotation and spatial-scaling transformation.

2. Convolution of the two normalized screens, giving the normalized Glass pattern.

3. Rotating and spatially scaling the normalized Glass pattern into its actual scale and orientation, as determined by the vectors $\mathbf{a}=\mathbf{f}_{1}-\mathbf{f}_{3}$ and $\mathbf{b}=\mathbf{f}_{2}-\mathbf{f}_{4}$, or, more explicitly, by Eqs. (21) and (22).

Note that the aim of the normalization is to bring all three functions in Eq. (27) to the same common basis (or coordinate system) before we perform the multiplication. The need for this normalization was more obvious in the periodic case (Propositions 2 and 3), where the entities to be multiplied in the spectral domain were impulse combs or impulse nailbeds, whose multiplication was done term by term; but it remains essential in the aperiodic case, too, although the entities to be multiplied are continuous.

This result can be stated more formally as the aperiodic counterpart of Proposition 10.5 of Ref. 3, p. 339; note that functions denoted by boldface letters indicate mappings of $\mathrm{R}^{2}$ onto itself:

Proposition 7. Let $r_{1}(\mathbf{x})$ and $r_{2}(\mathbf{x})$ be two aperiodic screens that are obtained by applying linear mappings (scalings, rotations, etc.) $\mathbf{g}_{1}(\mathbf{x})$ and $\mathbf{g}_{2}(\mathbf{x})$, respectively, on two isometric aperiodic screens that have intensity profiles $p_{1}\left(\mathbf{x}^{\prime}\right)$ and $p_{2}\left(\mathbf{x}^{\prime}\right)$ :

$$
r_{1}(\mathbf{x})=p_{1}\left(\mathbf{g}_{1}(\mathbf{x})\right), \quad r_{2}(\mathbf{x})=p_{2}\left(\mathbf{g}_{2}(\mathbf{x})\right) .
$$

Then the Glass pattern $m(\mathbf{x})$ in the superposition of $r_{1}(\mathbf{x})$ and $r_{2}(\mathbf{x})$ is given by

$$
m(\mathbf{x})=p(\mathbf{g}(\mathbf{x})),
$$

where

1. $p\left(\mathbf{x}^{\prime}\right)$, the normalized intensity profile of the Glass pattern, is the convolution of the normalized intensity profiles of the original screens:

$$
p\left(\mathbf{x}^{\prime}\right)=p_{1}\left(\mathbf{x}^{\prime}\right) * * p_{2}\left(-\mathbf{x}^{\prime}\right) ;
$$

2. $\mathbf{g}(\mathbf{x})$, the linear transformation that brings $p\left(\mathbf{x}^{\prime}\right)$ back into the actual scale and orientation of the Glass pattern $m(\mathbf{x})$, is given by

$$
\mathbf{g}(\mathbf{x})=\mathbf{g}_{1}(\mathbf{x})-\mathbf{g}_{2}(\mathbf{x})
$$

Note that if the explicit expressions of the linear mappings $\mathbf{g}_{1}(\mathbf{x})$ and $\mathbf{g}_{2}(\mathbf{x})$ are given by

$$
\begin{aligned}
& \mathbf{g}_{1}(\mathbf{x})=\left(\begin{array}{l}
u_{1} x+v_{1} y \\
u_{2} x+v_{2} y
\end{array}\right)=F_{1} \cdot \mathbf{x} \\
& \text { with } F_{1}=\left(\begin{array}{ll}
u_{1} & v_{1} \\
u_{2} & v_{2}
\end{array}\right)=\left(\begin{array}{l}
\mathbf{f}_{1} \\
\mathbf{f}_{2}
\end{array}\right), \quad \mathbf{x}=(x, y), \\
& \mathbf{g}_{2}(\mathbf{x})=\left(\begin{array}{l}
u_{3} x+v_{3} y \\
u_{4} x+v_{4} y
\end{array}\right)=F_{2} \cdot \mathbf{x} \\
& \text { with } F_{2}=\left(\begin{array}{ll}
u_{3} & v_{3} \\
u_{4} & v_{4}
\end{array}\right)=\left(\begin{array}{l}
\mathbf{f}_{3} \\
\mathbf{f}_{4}
\end{array}\right), \quad \mathbf{x}=(x, y),
\end{aligned}
$$

then we have

$$
\begin{aligned}
& \mathbf{g}(\mathbf{x})=\left(\begin{array}{l}
\left(u_{1}-u_{3}\right) x+\left(v_{1}-v_{3}\right) y \\
\left(u_{2}-u_{4}\right) x+\left(v_{2}-v_{4}\right) y
\end{array}\right)=F_{M} \cdot \mathbf{x} \\
& \text { with } F_{M}=\left(\begin{array}{ll}
u_{1}-u_{3} & v_{1}-v_{3} \\
u_{2}-u_{4} & v_{2}-v_{4}
\end{array}\right)=\left(\begin{array}{l}
\mathbf{a} \\
\mathbf{b}
\end{array}\right),
\end{aligned}
$$

where

$$
F_{M}=F_{1}-F_{2},
$$

namely,

$$
\left(\begin{array}{l}
\mathbf{a} \\
\mathbf{b}
\end{array}\right)=\left(\begin{array}{l}
\mathbf{f}_{1}-\mathbf{f}_{3} \\
\mathbf{f}_{2}-\mathbf{f}_{4}
\end{array}\right) .
$$

This indeed gives us, the connection between Eq. (30) and the basis vectors of the individual screens and their resulting moiré, which are, respectively, $\mathbf{f}_{1}$ and $\mathbf{f}_{2}, \mathbf{f}_{3}$ and $\mathbf{f}_{4}$, and $\mathbf{a}$ and $\mathbf{b}$, where $\mathbf{a}=\mathbf{f}_{1}-\mathbf{f}_{3}$ and $\mathbf{b}=\mathbf{f}_{2}-\mathbf{f}_{4}$, exactly as in the periodic case [see Eq. (16)]. ${ }^{14}$

Thus by extending our moiré theory from the $2 \mathrm{D}$ periodic case to the $2 \mathrm{D}$ aperiodic case, we have succeeded in extracting the isolated Glass pattern from the screen superposition both in the spectral domain and in the image domain. Let us now see the full significance of this result.

\section{B. Shape of the Glass Pattern}

Following our previous experience with periodic dot screens (see Subsection 2.D.1), we divide our study into three cases.

Case 1. Suppose, first, that one of the superposed layers is an aperiodic screen consisting of arbitrarily positioned dots having a fixed shape (such as the digit 1) and that the second layer is an aperiodic screen consisting of tiny pinholes on a black background, where the dot locations in the two screens are identical (or slightly transformed). In the convolution such pinholes play the role of very narrow pulses with amplitude 1 . According to Remark 2 in Subsection 3.B, the shape of the Glass pattern that is generated in the layer convolution is determined basically by the convolution of one element from each layer. As shown in Fig. 7(a), this means that in this case, just as happens in the superposition of periodic layers (Subsection 2.D), the moiré (or Glass) pattern that appears in the superposition is essentially a magnified and rotated version of an individual dot of the first screen. But as already explained in Remark 1 at the end of Subsection 3.A, the Glass pattern generated in the aperiodic case is not periodically repeated throughout the superposition, as in the periodic case, and it consists of only one copy of the magnified dot shape (compare Figs. 2 and 3). 
This surprising result seems at first to contradict the basic properties of Glass patterns as generally known until now. A classical Glass pattern is expected to be brighter in its center than in areas farther away, owing to the partial overlapping of the dots of the two layers in this area [see Fig. 1(a)]. But the Glass pattern of Fig. 2 seems to completely contradict this property.

In reality, however, there is no contradiction at all. The key point is that in classical Glass patterns, as known until now, the two superposed layers were identical, both consisting of black dots on a white background. But if, as shown in Fig. 2, one of the screens consists of tiny pinholes on a black background and the other screen has a fixed dot shape, then the convolution of the dot shape of one screen with the dot shape of the other screen, gives, indeed, a Glass pattern that has the intensity profile of the dot shape of the other screen.

Case 2. Suppose now that we replace our pinhole screen by an inverse-video copy of itself, consisting of tiny black dots on a white (or, rather, transparent) background. In this case the convolution of the individual dot shapes of the two layers basically gives an inverse-video version of the result in the first case, as shown in Fig. 7(b). Hence if one of the screens contains tiny black dots and the other screen has a fixed dot shape, then the moiré intensity profile that we obtain is a magnified version of the individual dot shape of the other screen, but this time in inverse video. In our example, we will obtain a single 1-shaped Glass pattern that is brighter inside the digit shape and darker outside. Note, however, that this moire intensity profile is weaker and less impressive than that of the previous case.

Case 3. Finally, when none of the superposed layers consists of tiny dots (either white or black), or when the superposed layers do not have fixed element shapes, the intensity profile form of the resulting moiré (or Glass pattern) is still a magnified version of the convolution of the dot shapes of the two layers. This convolution gives some kind of blending between the original dot shapes, but the resulting shape has a blurred or smoothed-out appearance resembling a 2D Gaussian, with no recognizable shapes. As we can now understand, this is exactly what happens in classical Glass patterns, where the two superposed layers are identical [as in Fig. 1(a)] or where their dot shapes are arbitrary. This is also the reason for which Glass patterns described in the past did not have the shape of a magnified element from one of the superposed layers.

\section{Orientation and Size of the Glass Pattern}

Looking at Figs. 2 and 3, we already know by now how to explain their common 1-shaped moiré (or Glass) pattern: In both the periodic and the aperiodic cases, this is simply a normalized convolution of a single pinhole from one layer and a single 1 element from the other layer. But how can we explain the fact that in the two cases the resulting moiré (or Glass) patterns have the same orientation and the same size?

As we have seen, the $(1,0,-1,0)$ moire in the periodic case and the Glass pattern in the aperiodic case are governed by the same basis vectors in the spectrum [Eq. (16)]:

$$
\begin{aligned}
& \mathbf{a}=\mathbf{f}_{1}-\mathbf{f}_{3}, \\
& \mathbf{b}=\mathbf{f}_{2}-\mathbf{f}_{4} .
\end{aligned}
$$

Although in the aperiodic case these vectors cannot be interpreted in terms of periodicities as in the periodic case, they still determine the orientation and the size of the resulting moiré effect. For example, when the two superposed screens are congruent (meaning that they have the same scaling or, in the periodic case, the same frequencies or periodicities), the basis vectors $\mathbf{a}$ and $\mathbf{b}$ are perpendicular to the bisectors of the angles formed between the frequency vectors $\mathbf{f}_{1}, \mathbf{f}_{3}$ and $\mathbf{f}_{2}, \mathbf{f}_{4}$. This means that the $(1,0,-1,0)$ moire (or the Glass pattern) are closely perpendicular to the original screens $r_{1}(x, y)$ and $r_{2}(x, y)$ (see Figs. 2 and 3). As for the size of the Glass (or moiré) patterns, they are obtained by multiplying the original screen element size by the spatial scaling (magnification) of the moiré. For example, if the length of each of the basis vectors $\mathbf{f}_{1} \ldots \mathbf{f}_{4}$ of the original screens is $f$ and the length of each of the two basis vectors $\mathbf{a}$ and $\mathbf{b}$ of the Glass (or moiré) pattern is $f_{M}=0.1 f$, then the size of the 1-shaped moiré in both Figs. 2 and 3 will be ten times the size of the element 1 in the original screen. But whereas in the periodic case the magnification rate can be also interpreted in terms of periods, $T_{M}=10 T$, in the aperiodic case the interpretation is done in terms of the scaling ratio, which is clearly the aperiodic counterpart of the periodic notion of frequency ratio [compare Eqs. (18) and (19) in the aperiodic case with Eqs. (7) and (8) in the periodic case].

We therefore obtain the following remarkable result:

Proposition 8. If the respective dot screens in the periodic case and in the aperiodic case consist of the same microstructure elements and they have the same angle difference $\alpha$ and the same scaling factors, then the resulting $(1,0,-1,0)$ moiré (or Glass) patterns in the two cases have the same shape, the same size, and the same orientation. The only difference is that in the aperiodic case only one such pattern is generated, whereas in the periodic case there appear infinitely many replicas, as already explained in Remark 1.

The above result is clearly illustrated in Figs. 2 and 3.

\section{Superposition of Uncorrelated Screens}

What would have happened now if the original screens $r_{1}(x, y)$ and $r_{2}(x, y)$ had not been isometric (or at least correlated) before undergoing the linear rotation and scaling transformations $g_{1}(x, y)$ and $g_{2}(x, y)$ ? It is well known that two uncorrelated screens do not generate a Glass pattern in their superposition. This can be explained, just as in the case of aperiodic gratings (Subsection 3.B), by using the fact that the convolution in Eq. (29) is the cross correlation of $p_{1}\left(\mathbf{x}^{\prime}\right)$ and $p_{2}\left(\mathbf{x}^{\prime}\right)$ :

$$
p_{1}\left(\mathbf{x}^{\prime}\right) \star \star p_{2}\left(\mathbf{x}^{\prime}\right)=p_{1}\left(\mathbf{x}^{\prime}\right) * * p_{2}\left(-\mathbf{x}^{\prime}\right) .
$$

Therefore, as we have already seen in Subsection 3.B, if the original screens are correlated, their cross correlation contains in its center a privileged area that corresponds to the Glass pattern. But when the original screens are not correlated, their cross correlation does not contain such a privileged area, and it simply fluctuates around 
the same mean level throughout. Hence no visible structure (Glass pattern) appears in the screen superposition.

\section{E. Discussion}

We have seen that the fundamental moiré relationship between the image domain and the spectral domain holds also in the generalized case where the superposed screens are no longer periodic. Note, however, that in the case of aperiodic screens the spectral considerations are much less intuitive, and we have obtained them by virtue of the insights that we have gained by analyzing the simpler cases in the previous sections.

But the importance of our results is not only in the theoretical generalization of the periodic moire theory into the case of aperiodic layers: Our results allow, apparently for the first time, the quantitative prediction of the intensity profile of the resulting Glass patterns; and furthermore, they open the way to the synthesis of Glass patterns that have any desired shapes and intensity profiles.

These results may find application in several scientific and technological disciplines. For example, they can be used in vision research; in physiological experiments such as form perception; and in the study of the human visual system, where classical Glass patterns have been used for a long time. ${ }^{2,15}$ Possible technological fields of application may include precision optical alignment, image registration, measurement of microscopic displacements, ${ }^{16}$ analogic magnifiers, and even document security and authentication. ${ }^{17}$

\section{HIGHER-ORDER MOIRÉS}

It is interesting to note that the moiré theory that we have developed here for aperiodic line gratings and for aperiodic dot screens is in fact a generalization of the first-order $(1,-1)$ moiré between periodic line gratings or its $2 \mathrm{D}$ counterpart, the $(1,0,-1,0)$ moiré between periodic dot screens. The reason is that in aperiodic structures, higher-order moirés simply do not exist: Whereas in periodic cases new higher-order moirés may occur when a scaling ratio of $s=2,3$, etc., is applied to one of the superposed layers, in aperiodic cases no higher-order moirés can exist, since at such scaling ratios no correlation exists between the superposed layers [for instance, a random screen $r(x, y)$ is not correlated with $r(2 x, 2 y)$ ].

Furthermore, it turns out that even the other firstorder moirés from the periodic case do not have equivalents in the aperiodic case: As we have seen throughout this paper, our generalization from the periodic to the aperiodic case concerns only the subtractive $(1,-1)$ moire (or its $2 \mathrm{D}$ counterpart, the $(1,0,-1,0)$ moiré, in the case of dot screens) but not the additive $(1,1)$ moiré (or its $2 \mathrm{D}$ counterpart, the $(1,0,1,0)$ moiré). The aperiodic equivalent of the $(1,1)$ moiré would be obtained theoretically as a continuous extension of $d_{n}=a_{n}^{(1)} a_{n}^{(2)}$, namely, $P(n)$ $=P_{1}(n) P_{2}(n)$ [compare with Eqs. (13) and (24)]. But owing to the lack of inversion in $P_{2}(n)$, the result in the image domain is the cross correlation of $p_{1}\left(x^{\prime}\right)$ and $p_{2}\left(-x^{\prime}\right)$, which are not correlated [the correlated functions are $p_{1}\left(x^{\prime}\right)$ and $\left.p_{2}\left(x^{\prime}\right)\right]$. Therefore in such cases no Glass pattern is generated in the superposition.
The subtractive $(1,-1)$ moiré [and its $2 \mathrm{D}$ counterpart, the $(1,0,-1,0)$-moiré] are indeed the strongest, the simplest, and the most frequent of all the $\left(k_{1}, k_{2}\right)$ or $\left(k_{1}, k_{2}, k_{3}, k_{4}\right)$ moirés between periodic gratings (or dot screens). They give the most spectacular moiré effects between periodic layers, like those of Fig. 3, and their explanation, both in the image domain and in the spectral domain, is the most straightforward (see Chap. 4 of Ref. $3)$. It is therefore interesting to see that when we randomize the element locations in two originally periodic layers, the only moire that survives in the resulting layer superposition is precisely the $(1,-1)$ moire (in the case of gratings) or the (1, 0, - 1, 0) moiré (in the case of screens). Note that even in this surviving moiré, only one of the infinitely many moiré replicas from the periodic case still survives in the aperiodic case; it has been shown ${ }^{6}$ that this is precisely the one that is generated around the fixed point of the two superposed screens.

\section{CONCLUSIONS}

In spite of their different appearance, moiré effects that occur between periodic or between aperiodic structures are particular cases of the same basic phenomenon, and all of them satisfy the same fundamental rules. Superpositions of periodic structures are simply a particular case in which the elements within each of the layers are arranged periodically so the resulting moiré effect is periodic, too.

We have shown that Glass patterns between aperiodic layers can be analyzed by using an extension of the mathematical theory that governs the classical moiré patterns between periodic layers. Spectral-domain considerations are extended in a natural way to such aperiodic cases, although their handling is less intuitive than in the periodic case. Our results allow us to predict quantitatively the intensity profile of the resulting Glass patterns; furthermore, they lead us to the possibility of synthesizing Glass patterns that have any desired shapes and intensity profiles.

It should be noted that although we have limited our discussion here to monochrome black-and-white layers, the extension of our results to the multichromatic case is straightforward, and it can be done exactly as in the periodic case (see, for example, Chap. 9 in Ref. 3).

\section{APPENDIX A: INTERMEDIATE, PARTLY RANDOM CASES}

Having developed our generalized Fourier-based approach, which explains quantitatively the moiré (or Glass) patterns between both periodic and aperiodic, random layers, it would be interesting to see now whether this general theory could also predict the behavior of moiré patterns in intermediate cases, that is, when the element locations in the two superposed layers are neither fully periodic nor fully random. Such partly random layers can be obtained, for example, by adding a varying degree of randomness to the element locations in an initially fully periodic layer. As we can see in Fig. 15, the resulting Glass patterns in such cases indeed have an intermediate look: Depending on the degree of randomness be- 

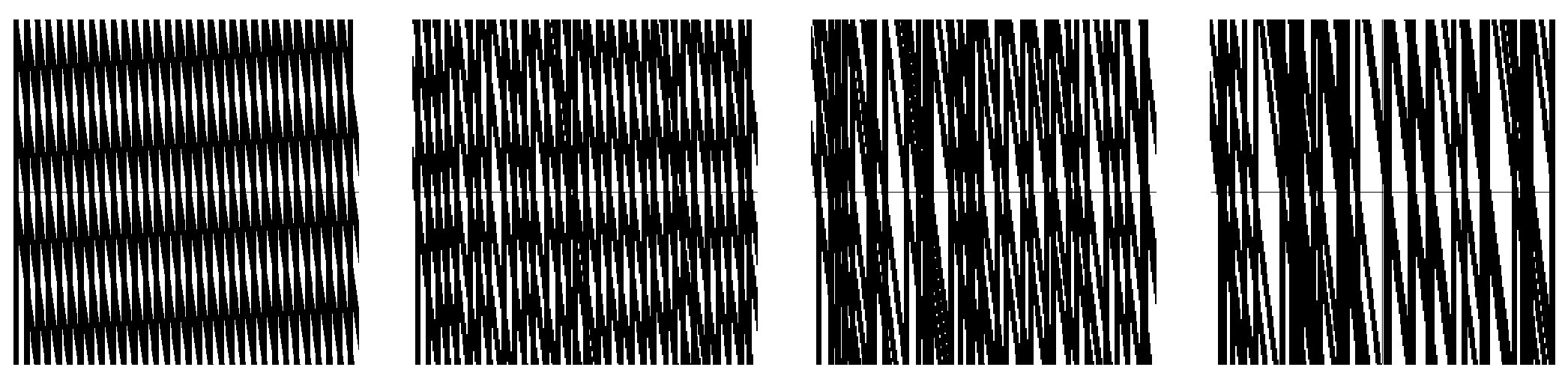

0
0
$\infty$
0
0
0
0
0
0
0
0
0
0
0
0
0
0
0
0
$z$
0
0
0
0
0
0
0
0
0
0
0
0
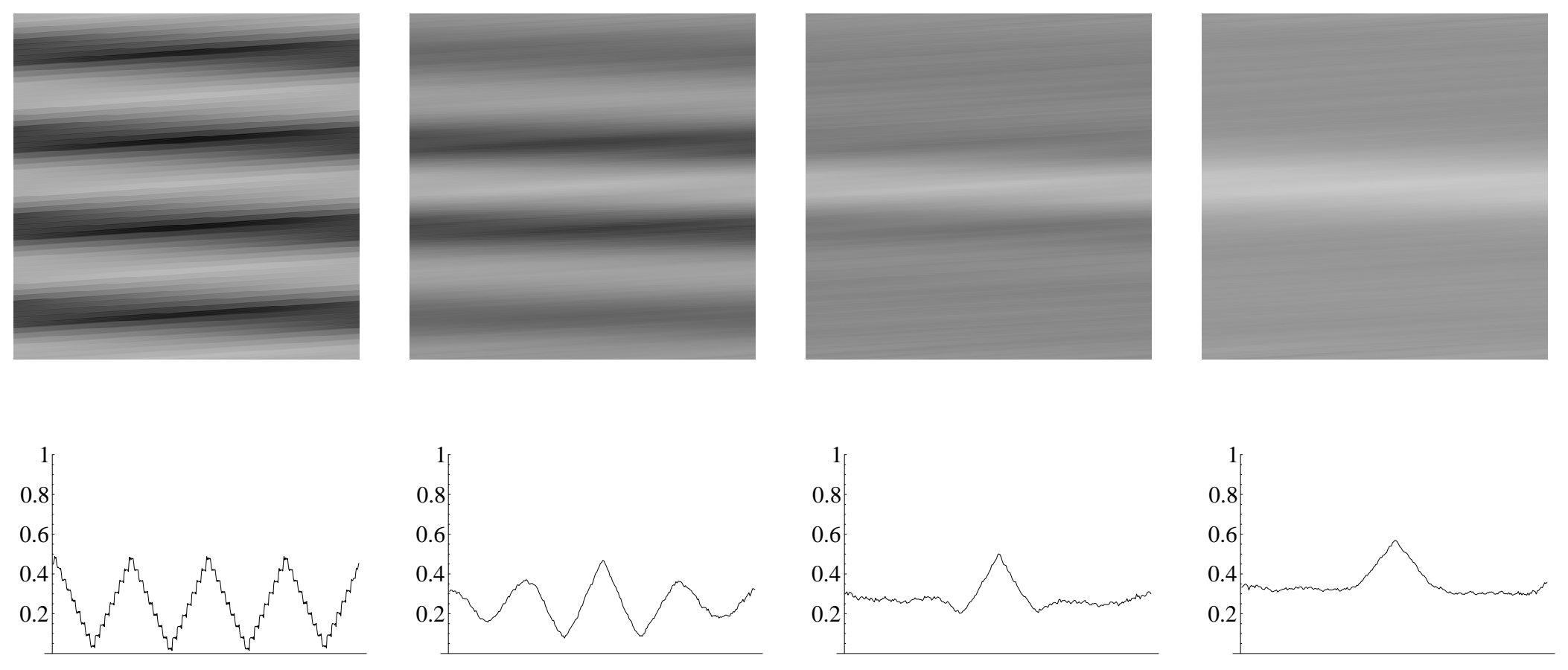

Fig. 15. Top row: a series of grating superpositions with a gradually increasing degree of randomness in the element locations. Note the increased fading out of the moiré oscillations as we go from the left (fully periodic) to the right (fully aperiodic or random). Center row: extracted moiré profiles, as obtained by inverse Fourier transform of the moiré blade extracted from the spectrum of the corresponding grating superpositions. Bottom row: cross sections through the extracted moiré profiles. Note the agreement between these calculated cross sections and the moiré profiles that are visible in the corresponding grating superpositions. 
ing added, they may have around the center one or more oscillations between darker and brighter areas. But because the correlation between the layers decreases with the distance, these oscillations are not repeated ad infinitum as in the periodic case, and they gradually fade out and disappear as we go farther from the center of the Glass pattern. Clearly, the higher the degree of randomness, the lower the number of oscillations around the center. These oscillations are, in fact, replicas of the central Glass pattern that gradually become more and more noisy as we go farther from the center, until they finally disappear completely within the background noise.

From the image-domain point of view, the center of the Glass patterns in Fig. 15 consists of a gray level brighter than areas farther away, owing to the partial overlapping of the microstructure elements of the two layers in this area. Because of the residual periodicity in the superposed layers, elements from the two layers half a period away from the center are more likely to fall side by side, thus increasing the covering rate and the macroscopic gray level. Depending on the degree of periodicity in the superposed layers, several such oscillations between brighter and darker areas may occur in the superposition. But as we go farther away from the center, the correlation between the two layers becomes lower, and the elements from both layers start falling in an arbitrary, noncorrelated manner; in this area the Glass pattern fades out, and we obtain a mean gray level that remains constant throughout.

Indeed, these image-domain considerations are fully confirmed in the spectral domain, too. As one would expect, the Fourier spectrum of such partly periodic layers is intermediate between the spectra of periodic and aperiodic cases: Its blade consists of partly blurred impulses corresponding to the frequencies in question plus a diffuse background that is typical of stochastic cases. Moreover, as shown in Fig. 15, the moiré intensity profile obtained by an inverse Fourier transform of the extracted moiré blade shows clearly the oscillations between darker and brighter areas, which fade out and disappear as we go farther away from the center; and the number of oscillations clearly depends on the degree of randomness. This confirms that our generalized Fourier-based approach indeed predicts the quantitative behavior of moiré patterns in all cases-periodic, random, and intermediate.

It should be noted that although the case of partly random layers has been illustrated here by line gratings for the sake of simplicity, the same results remain true for dot screens, too, and in particular for all the special cases enumerated in Subsection 4.B. For example, partly randomized versions of Fig. 3 indeed look intermediate between Figs. 2 and 3, with a sharp Glass pattern in the center that is surrounded by several blurred replicas depending on the degree of periodicity in the superposed layers.

\section{REFERENCES AND NOTES}

1. L. Glass, "Moiré effect from random dots," Nature 223, 578-580 (1969).

2. L. Glass and R. Pérez, "Perception of random dot interference patterns," Nature 246, 360-362 (1973).

3. I. Amidror, The Theory of the Moiré Phenomenon (Kluwer Academic, Dordrecht, The Netherlands, 2000).

4. I. Amidror, "Glass patterns and moiré intensity profiles: new surprising results," Opt. Lett. 28, 7-9 (2003).

5. http://lspwww.epfl.ch/books/moire/kit.html.

6. I. Amidror, "A unified approach for the explanation of stochastic and periodic moirés," J. Electron. Imaging (to be published).

7. I. Amidror, "Glass patterns in the superposition of random line gratings," J. Opt. A, Pure Appl. Opt. 5, 205-215 (2003)

8. R. N. Bracewell, The Fourier Transform and Its Applications, 2nd ed. (McGraw-Hill, Reading, N.Y., 1986).

9. R. N. Bracewell, Two Dimensional Imaging (Prentice-Hall, Englewood Cliffs, N.J., 1995).

10. Y. Nishijima and G. Oster, "Moiré patterns: their application to refractive index and refractive index gradient measurements," J. Opt. Soc. Am. 54, 1-5 (1964).

11. Note that this impulse is generated in the convolution by the $\left(k_{1}, k_{2}\right)$ impulse in the spectrum $R_{1}(u, v)$ of the first image and the $\left(k_{3}, k_{4}\right)$ impulse in the spectrum $R_{2}(u, v)$ of the second image.

12. A. Rosenfeld and A. C. Kak, Digital Picture Processing, Vol. 1, 2nd ed. (Academic, Boca Raton, Fla., 1982).

13. J. D. Gaskill, Linear Systems, Fourier Transforms, and Optics (Wiley, New York, 1978).

14. It is interesting to note that just like its periodic counterpart (see Sec. 10.9 of Ref. 3), this proposition remains true for nonlinear transformations $g_{i}(x, y)$, too, i.e., when the original aperiodic layers undergo any given geometric transformations. In such cases, part 2 of the proposition simply gives the geometric transformation that is undergone by the resulting Glass pattern.

15. S. C. Dakin, "The detection of structure in Glass patterns: psychophysics and computational models," Vision Res. 37, 2227-2246 (1997).

16. K. Patorski, Handbook of the Moiré Fringe Technique (Elsevier, Amsterdam, 1993), pp. 99-139.

17. I. Amidror, "A new print-based security strategy for the protection of valuable documents and products using moiré intensity profiles," in Optical Security and Counterfeit Deterrence Techniques IV, R. L. Van Renesse, ed., Proc. SPIE 4677, 89-100 (2002). 